\title{
Review
}

\section{Electromagnetic wave propagation in rain and polarization effects}

\author{
By Sogo OKAMURA ${ }^{* 1, * 2, \dagger}$ and Tomohiro OGUCHI ${ }^{* 3}$
}

(Contributed by Sogo Okamura, M.J.A.)

\begin{abstract}
This paper summarizes our study on microwave and millimeter-wave propagation in rain with special emphasis on the effects of polarization. Starting from a recount of our past findings, we will discuss developments with these and how they are connected with subsequent research.
\end{abstract}

Keywords: radiowave propagation, attenuation by rain, polarization effects, radar meteorology, multiple scattering effects

\section{Introduction}

In the middle of the $1950 \mathrm{~s}$, a feasibility study on using the millimeter-wave band in radio communications started at the Radio Research Laboratory (currently the National Institute of Information and Communications Technology) to meet the everincreasing demand for frequency resources. In 1956, the first author of this paper assumed a concurrent position as Chief of the Millimeter Wave Research Section of that laboratory, and initiated research on millimeter-wave propagation in rain in the $35-\mathrm{GHz}$ band.

Radiowave attenuation due to rain was anticipated to be increasingly severe for frequencies higher than about $10 \mathrm{GHz}$. The theoretical work by Ryde and Ryde ${ }^{1)-3)}$ in the early 1940s represents seminal research in this field. They calculated the electromagnetic cross sections of spherical raindrops, without restrictions in the ratio of drop size to wavelength (Mie scattering regime), and, using microphysical parameters of rain, derived the relation between rainfall rate and attenuation. The experimental results obtained back then have generally confirmed the predictions. ${ }^{4), 5)}$

\footnotetext{
*1 Professor Emeritus, The University of Tokyo, Tokyo, Japan.

*2 The Japan Academy, Tokyo, Japan.

*3 Institute of Science and Technology, Kanto Gakuin University, Yokohama, Japan.

$\dagger \quad$ Correspondence should be addressed: S. Okamura, 4-12-15 Numabukuro, Nakano-ku, Tokyo 165-0025, Japan (e-mail: ysokamu@poem.ocn.ne.jp).
}

We started measurements to reexamine the thenpublished data. During the course of these measurements, we were confronted with a rather peculiar phenomenon, i.e., the attenuation in horizontal polarization tended to be slightly larger than that in vertical polarization. ${ }^{6}$ ) To the best of the authors' knowledge, this was the first work that explicitly demonstrated the effects of polarization on attenuation. Distortion in raindrops was suspected to be the cause of this difference. In fact, the photographic measurements of water drops falling at terminal velocity in air revealed that the drops were not spherical but asymmetrical about the horizontal plane, generally with a flattened base for large drops. ${ }^{7}$ ) The dependence of rain attenuation on polarization was theoretically estimated by taking into account drop distortion, assuming there was little deviation from the spherical shape. ${ }^{8)}$ Within the limits of perturbation calculations, the agreement between measured and estimated values was satisfactory. ${ }^{6}$ )

Although the effect of polarization was found to be small, this has later become of practical importance, because terrestrial and earth-space communication systems have used two orthogonal polarizations at an identical frequency to increase the capacity of communications; the coupling between two orthogonal polarization channels, if it occurs, will greatly limit the efficient use of these orthogonal channels. This really is the cause for nonspherical raindrops falling with trajectories that are not in parallel with the direction of reference polarization. Very many theoretical and experimental studies were conducted on this in the 1970 s in various countries. 
Meteorological radars were initially operated on a single polarization basis. However, it was observed that the properties of polarization are important in radar meteorology to identify hydrometeors and to accurately monitor rain parameters. In fact, Atlas et al. pointed out the importance of the polarization properties of hydrometeors in 1953, based on approximate theory for small spheroids. ${ }^{9)}$ Since then, meteorological radars for microphysical research have been partially employing dual-polarization capabilities, and fully polarimetric weather radars are now being used in several countries. There has been a tendency for even operational weather-forecast agencies to adopt dual-polarization capabilities to obtain better forecasts of very localized and intense rainfall.

This paper is organized as follows. Section 2 describes the general procedure for calculating the relation between rainfall rate and attenuation. Section 3 outlines the way we did attenuation measurements in the period from around the end of the $1950 \mathrm{~s}$ to the beginning of the $1960 \mathrm{~s}$, and how attenuation in horizontal polarization differs from that in vertical polarization. The procedure for calculating the scattering properties of distorted raindrops, and the major calculated results are presented in Section 4. Section 5 discusses the interference between two orthogonal polarization channels due to the depolarization effects of rain on dual-polarization communication systems, and how we could eliminate this interference. Section 6 outlines the advantages of employing dual-polarization over single polarization in radar meteorology, and presents some of the outcomes that resulted from using dual-polarization capabilities. The discussion thus far is based on the single-scattering regime. How multiple scattering affects attenuation and depolarization has been an unanswered question of long standing. Section 7 is devoted to this problem, and briefly outlines our findings on the effects of multiple scattering in rain. Finally, Section 8 of this paper draws some of the conclusions we reached. (A review of the research in this field up to around 1983 was presented by Oguchi. ${ }^{10)}$ )

\section{Scattering amplitude of raindrops and attenuation by rain}

This section first outlines the general procedure for calculating the attenuation and phase shift of electromagnetic waves traveling in a rain-filled medium. Then, assuming that the raindrops are spheres, we will give estimated attenuation values as a function of the frequency to find the significance of rain effects in the microwave and millimeter-wave regions.

When a plane electromagnetic wave with polarization vector $\hat{\mathbf{e}}$ is incident on a raindrop at the origin, it induces a transmitted field in the interior of the drop and a scattered field. Assuming that the incident wave with unit amplitude and frequency $f$ is propagating in direction $\hat{\mathbf{K}}_{1}$, the scattered electric field, $\mathbf{E}^{s}$, toward direction $\hat{\mathbf{K}}_{2}$ may be written in the far-field region as:

$$
\mathbf{E}^{s}=\mathbf{f}\left(\hat{\mathbf{K}}_{1}, \hat{\mathbf{K}}_{2}\right) r^{-1} \exp \left(-i k_{0} r\right)
$$

where vector function $\mathbf{f}\left(\hat{\mathbf{K}}_{1}, \hat{\mathbf{K}}_{2}\right)$, often referred to as "scattering amplitude", denotes the amplitude, phase, and the state of polarization of the scattered field, $r$ is the distance from the origin to the observation point, and $k_{0}$ is the free-space propagation constant defined by $2 \pi f / c_{0}$, where $c_{0}$ is the speed of light in free space. An $\exp (+i 2 \pi f t)$ time convention is assumed and is suppressed.

The propagation constant, $k$, in a space containing many raindrops is given by ${ }^{11)}$

$$
k=k_{0}+\frac{2 \pi}{k_{0}} \int \hat{\mathbf{e}} \cdot \mathbf{f}\left(\hat{\mathbf{K}}_{1}, \hat{\mathbf{K}}_{1}, a\right) n(a) d a
$$

where $\mathbf{f}\left(\hat{\mathbf{K}}_{1}, \hat{\mathbf{K}}_{1}, a\right)$ denotes the forward scattering amplitude of a raindrop with radius $a$, and $n(a) d a$ is the number of drops per cubic meter in space with radius $a$ in range $d a$ and is a function of rainfall rate $R$. (Note that the discussion in Sections $2-6$ is based on the single-scattering approximation.) Since the propagation factor in a rain medium is $\exp (-i k r)$, the real and imaginary parts of $k$ are respectively responsible for the phase shift and attenuation of the wave propagating in the rain medium.

In the attenuation measurements, the attenuation, $A$, is often given in $\mathrm{dB} / \mathrm{km}$ and is written in the following form:

$$
A=4.343 \times 10^{3} \times \int Q_{t}(a) n(a) d a
$$

where $Q_{t}$, commonly termed the "total cross section", is the sum of the power scattered in all directions from a raindrop and the power absorbed in the drop, when it is radiated by a plane wave of unit power per unit area. There is a well-known relation between $Q_{t}$ and the scattering amplitude, $\mathbf{f}$, in the forward direction $^{12)}$

$$
Q_{t}=-\left(4 \pi / k_{0}\right) \operatorname{Im}\left[\hat{\mathbf{e}} \cdot \mathbf{f}\left(\hat{\mathbf{K}}_{1}, \hat{\mathbf{K}}_{1}\right)\right] .
$$

Naturally, the attenuation formula obtained from the imaginary part of the propagation constant, $k$, agrees with Eq. [3], when Eq. [4] is referred to. 
The scattering amplitude, $\mathbf{f}$, is obtained by solving a boundary-value problem at the surface of a raindrop. If the drop is spherical in shape, there are analytic solutions to the boundary-value problem (Mie-Stratton expression ${ }^{13)}$ ). However, numerical computations of this expression, containing complex arithmetic, were laborious and time consuming in the pre-computer age of Ryde and Ryde. ${ }^{1)-3)}$ The permittivity of water in the microwave and millimeter-wave regions has a relatively high loss component, hence elementary vector solutions to the field inside a raindrop contain spherical Bessel functions with complex arguments.

Drop-size distribution $n(a)$ can be obtained by measuring the size distribution of raindrops reaching the ground, and converting it to a distribution in space with the aid of the fall velocity of raindrops. The drop-size distribution is a function of the rainfall rate, and depends on rain types. Although the measurements were made more than sixty years ago, the distribution by Laws and Parsons, ${ }^{14)}$ among others, is considered to be typical of the average distribution both for widespread rain (in the lower rainfall-rate range) and for convective rainfall (in the higher rainfall-rate range). Therefore, we have mostly used this size distribution in our theoretical work. Figure 1 plots the Laws and Parsons size distribution (thick solid curves), as well as the negative exponential relation proposed by Marshall and Palmer (fine solid lines). ${ }^{15)}$ The rainfall rate, $R$, in millimeters per hour is related to both drop-size distribution and fall velocity expressed by

$$
R=4.8 \pi \times 10^{-3} \times \int v(a) a^{3} n(a) d a
$$

where $v(a)$ is the terminal fall velocity in meters per second and $a$ is the drop radius in millimeters.

There is an example of the calculated attenuation values in Fig. 2 as a function of the frequency, both for Laws and Parsons (solid curves) and for Marshall and Palmer (dashed curves) size distributions. ${ }^{16)}$ Note that the attenuation tends to be very high as frequency approaches several tens of gigahertz, and is maximum at about $100 \mathrm{GHz}$. Further increases in frequency decrease the attenuation slightly so that it approaches the asymptotic value in the optical limit.

\section{Attenuation measurements}

This section summarizes the attenuation measurements that we did during the period from around the end of the $1950 \mathrm{~s}$ to the beginning of the $1960 \mathrm{~s}$,

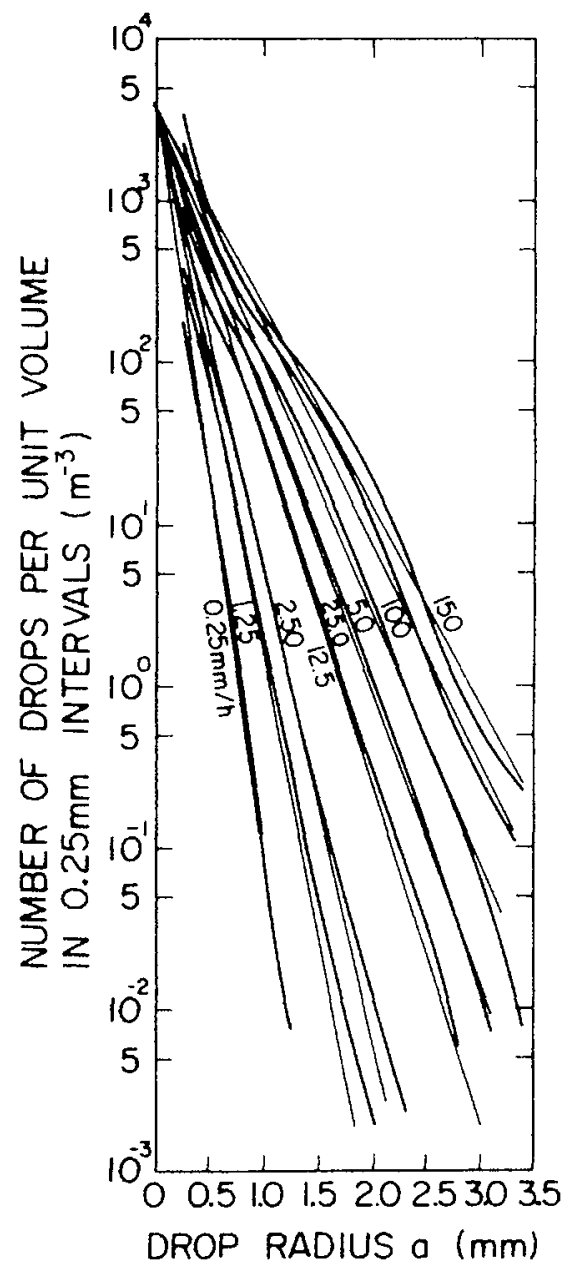

Fig. 1. Raindrop-size distributions in space. Parameters are rainfall rates. Thick solid curves are derived from measurements by Laws and Parsons, ${ }^{14)}$ and the fine solid lines are the negative exponential size distributions proposed by Marshall and Palmer. $^{15)}$ (After Oguchi. ${ }^{10)}$ (C) 1983 IEEE.)

and how attenuation in horizontal polarization differs from that in vertical polarization.

The equipment used in the attenuation measurements was a frequency-modulated radar operating at $\left.34.8 \mathrm{GHz},{ }^{17}\right)$ similar to that used by Crawford and Hogg of Bell Laboratories. ${ }^{18)}$ The transmitter-andreceiver polarization could be changed by replacing the short straight-waveguide section, connected just behind the circular primary horn, by using a $90^{\circ}$ twist section. A trihedral corner reflector with a crosssectional area of $1.7 \mathrm{~m}^{2}$ was located on the top of a building $400 \mathrm{~m}$ from the radar site. A very simple rain gauge was placed at the radar site. A rain collection funnel with a diameter of $0.35 \mathrm{~m}$ was attached to a graduated cylinder, and the rise in the water level 


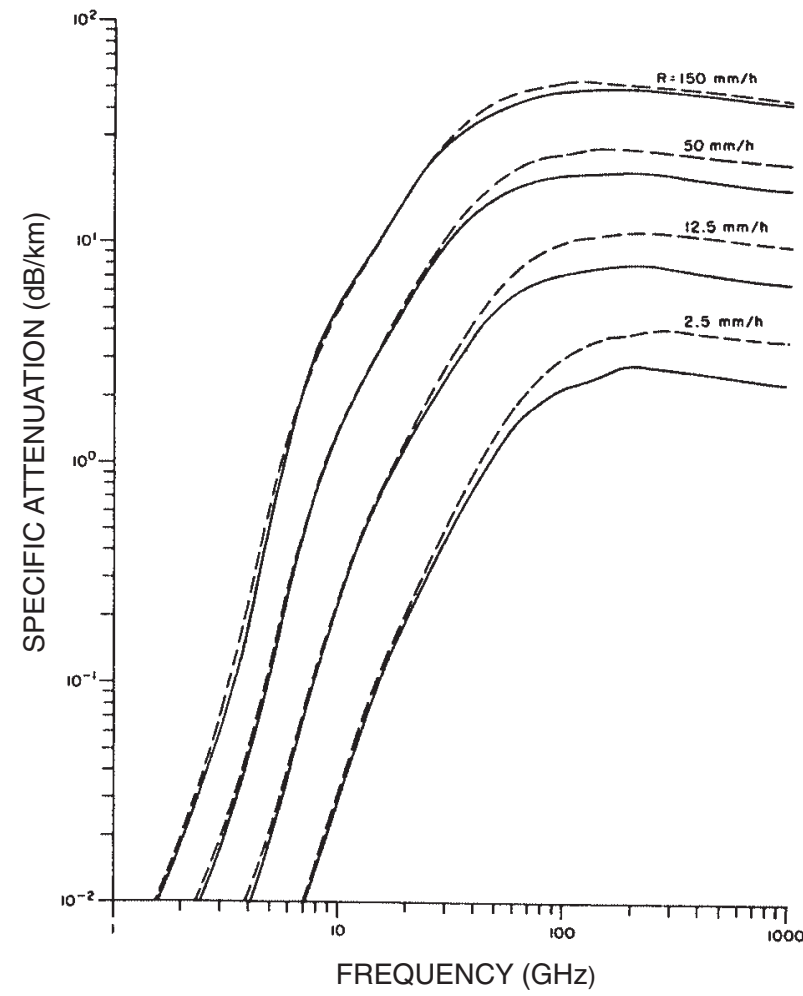

Fig. 2. Calculated rain attenuation versus frequency at water temperature of $20^{\circ} \mathrm{C}$. Solid curves are for Laws and Parsons size distributions and dashed curves are for Marshall and Palmer size distributions. (After Rogers and Olsen. ${ }^{16)}$ )

was measured every $30 \mathrm{sec}$ or $1 \mathrm{~min}$, depending on the rain intensity. The measured values were then converted into corresponding values in $\mathrm{mm} / \mathrm{h}$ units. Preliminary measurements were made during heavy rainfall, first in vertical polarization, and then in horizontal polarization. Figure 3 has the scatter plots of attenuation as a function of the rainfall rate in (a) vertical and (b) horizontal polarizations. The two solid lines are the results of the least-squares fitting to data points, and the dashed lines are the theoretical predictions made by Ryde and Ryde. ${ }^{1-3)}$ Although the measured values agreed favorably with the theoretical ones when the incident-wave polarization was horizontal, the attenuation in vertical polarization was in some degree lower than the theoretical prediction. There was some ambiguity as to whether attenuation really depended on polarization, since the measurements were made at different times or even on different days.

To overcome this problem, the waveguide section just behind the circular primary horn was replaced by a ferrite switch that rotated the polarization by $90^{\circ}$ when the exciting current was on. The
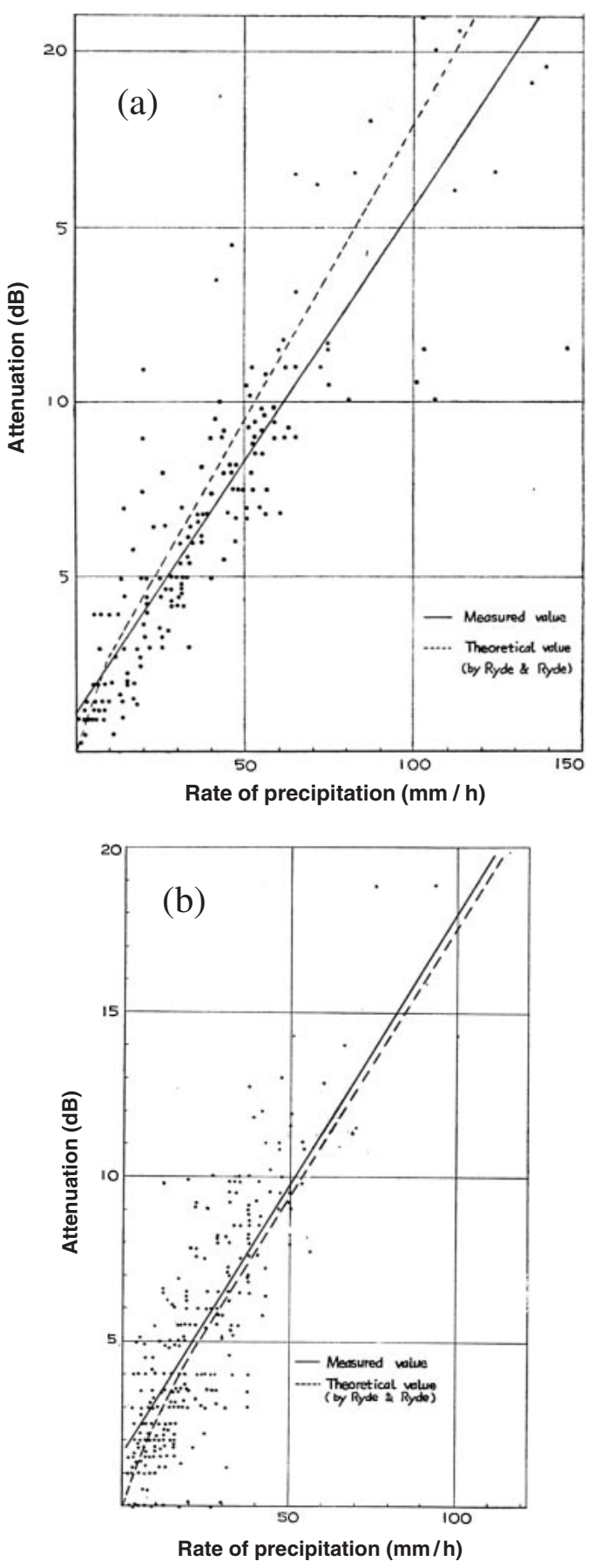

Fig. 3. Attenuation versus rainfall rate at $34.8 \mathrm{GHz}$ : (a) Vertical polarization and (b) horizontal polarization. ${ }^{17)}$ Solid lines are those obtained by least squares fitting to data points. Dashed lines are the theoretical predictions by Ryde and Ryde. ${ }^{1)-3)}$

rain gauge was also replaced by a fast-response tipping bucket gauge with a funnel diameter of $0.67 \mathrm{~m}$. Attenuation was then measured along a 3.55- 


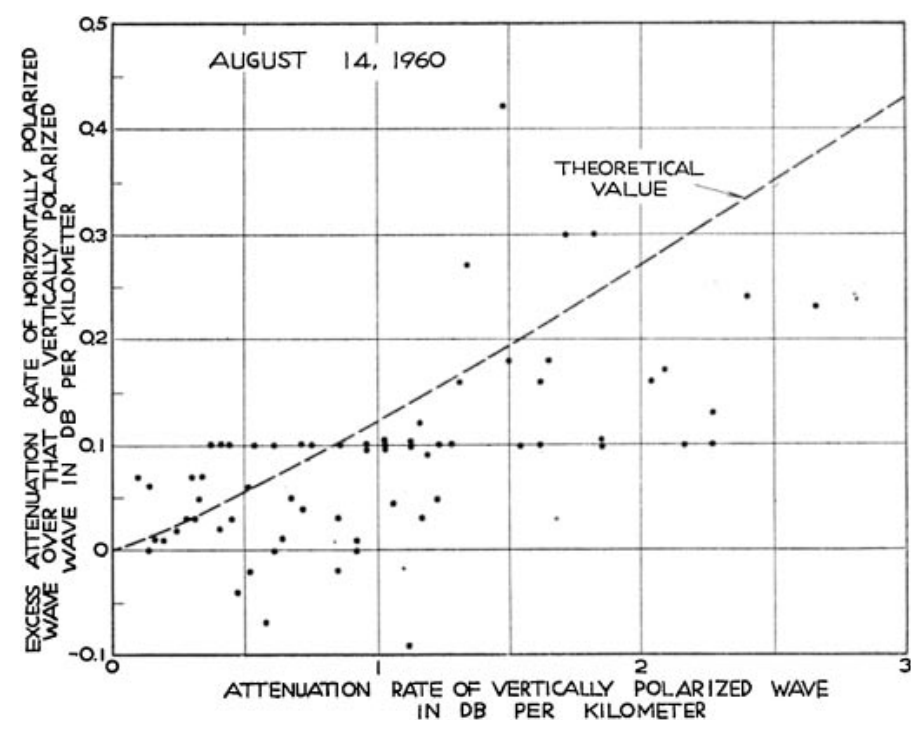

Fig. 4. Scatter plots of attenuation differences between horizontal and vertical polarizations as a function of attenuation in vertical polarization $\left.(\mathrm{dB} / \mathrm{km}){ }^{6}\right)$ Attenuation difference is defined as the attenuation in horizontal polarization $(\mathrm{dB} / \mathrm{km})$ minus the attenuation in vertical polarization $(\mathrm{dB} / \mathrm{km})$. Dashed curves are the theoretical estimates obtained by perturbation calculations. ${ }^{8)}$

km propagation path under showery conditions, by changing the polarization state from vertical to horizontal, and vice versa, in 5 -minute intervals. ${ }^{6)}$ Figure 4 has the scatter plots of the difference in attenuation as a function of attenuation in vertical polarization. The difference in attenuation was defined as the attenuation in horizontal polarization $(\mathrm{dB} / \mathrm{km})$ minus the attenuation in vertical polarization $(\mathrm{dB} / \mathrm{km})$. Instead of using the rainfall rate, we used attenuation as the abscissa because it is a more reliable measure of the path-averaged rainfall rate. From that experimental evidence, we had a firm belief that attenuation really depended on polarization. To the best of the authors' knowledge, these were the first measurements that explicitly demonstrated the effects of polarization on attenuation. This phenomenon has later come to light, since in various branches of radio communications through rain and in radar meteorology, we have had to encounter not just minor advantages and disadvantages of the effect of polarization, as will be described in the later sections. The difference in attenuation was later confirmed by Semplak's measurements at $30.9 \mathrm{GHz}^{19)}$

The distortion of raindrops from a spherical shape was suspected as the reason for the difference in attenuation. In fact, Magono measured the shape of water drops falling at terminal velocity in stagnant air, and he found that the drops were not spherical but asymmetrical about a horizontal plane, generally with a flattened base for large drops. ${ }^{7)}$ This motivated us to start a rather lengthy course of theoretical investigations into scattering from nonspherical raindrops, as will be described in the next section. The dashed lines in Fig. 4 are the results of perturbation calculations made under the assumption that raindrops did not deviate much from their spherical shape. ${ }^{8)}$

\section{Shape and scattering properties of distorted raindrops}

4.1. Shape of raindrops. The shape of raindrops was initially studied from a meteorological point of view, since it was relevant to various subjects in meteorology, such as fall velocity and drop breakup. Many investigators have made photographic measurements of the drop shape. Some measurements were made while the drops were suspended in the air stream of a vertical wind tunnel, and some were made when the drops attained their terminal velocity after falling from a sufficient height $(\sim 12 \mathrm{~m})$ in stagnant air. These measurements revealed that water drops larger than about $1.0 \mathrm{~mm}$ in radius were of oblate spheroidal shape with a flattened base.

Some other investigators have theoretically studied the drop shape, by solving an equation describing the balance of internal and external pressure at the surface of a drop. Because it has been difficult to solve this equation rigorously, several 


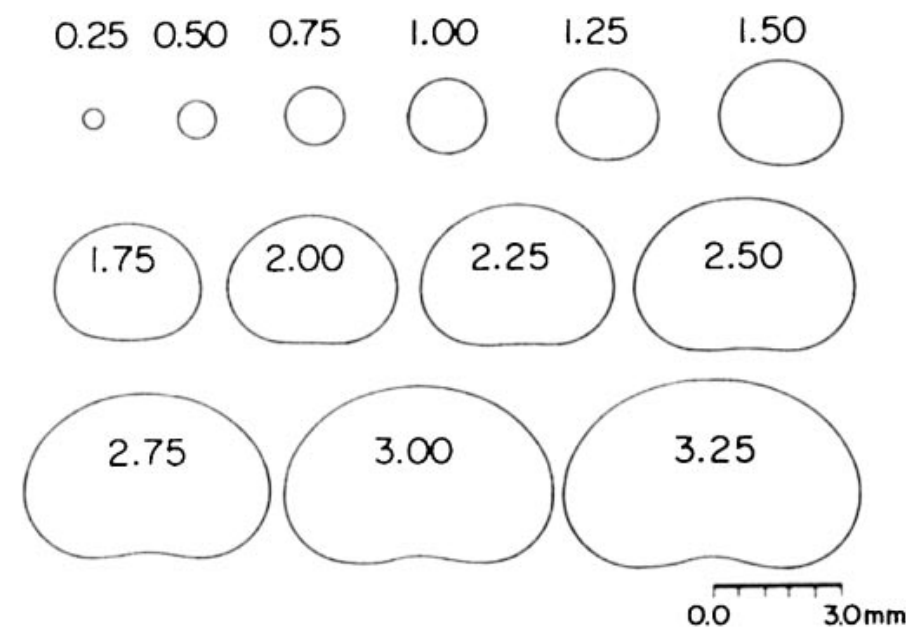

Fig. 5. Shapes of drops falling in stagnant air calculated by the procedure in Ref. 20. The numbers indicate the equivolume sphere radius. (After Oguchi. ${ }^{10)}$ C 1983 IEEE.)

assumptions or approximations have had to be introduced. Pruppacher and Pitter introduced some assumptions into the aerodynamic pressure around the surface of a water sphere, and numerically solved the pressure-balance equation. ${ }^{20)}$ Figure 5 shows the calculated drop shapes of 13 water drops whose equivolume radii range from 0.25 to $3.25 \mathrm{~mm}$. The shapes agreed reasonably well with those derived from measurements in those days. Note that the study of drop shape is again in the spotlight today, in connection with accurate measurements of rainfall that are possible due to the advent of polarimetric weather radars, which we shall discuss in Section 6 . Recent measurements of drop shapes by two-dimensional video disdrometers under calm-air conditions seem to indicate that the Pruppacher-Pitter model slightly overestimates real drop deformation; no concave deformation of the bottom of large drops has yet been found. ${ }^{21)-23)}$ (Video disdrometers are optical instruments that can measure the drop sizes, shapes, and numbers of drops per unit area falling in unit time.) In our theoretical treatment and numerical calculations of scattering properties, we initially used an oblate spheroidal model and then the Pruppacher-Pitter model.

4.2. Procedure for calculating scattering properties of distorted raindrops. Around 1958, we started theoretical investigations to account for the effects of polarization, as described in Section 3. Because the measurements were made at $34.8 \mathrm{GHz}$, the size of large raindrops was almost equal to the wavelength $(\sim 8.6 \mathrm{~mm})$. There are no analytic solutions to distorted drops. When there is not much distortion from the shape of a sphere, we may evaluate an additional scattering term (perturbation term) by using a perturbation calculation. The addition of this term to the sphere-scattering term may be an approximate solution to the problem. There are generally no restrictions on the drop size in this technique, except that there is not much distortion from the shape of a sphere. In 1956, Mushiake developed a perturbation theory for scattering from perfectly conducting spheroids with small eccentricity. ${ }^{24)}$ We extended his technique to the scattering from lossy dielectric spheroids. ${ }^{8)} \mathrm{We}$ tentatively evaluated the first-order perturbation term assuming that the drop shape was spheroidal. Because of the perturbation calculation, the scattering amplitude for large drops contained some error. In the first stage of numerical evaluation, we used a hand calculator, or an electric-powered calculator to calculate the spherical Bessel functions with complex arguments. The main part of the computation was done by a fully transistorized electronic computer that had just commenced operation at the Electrical Engineering Department of Keio University.

Let us assume, for a while, that the drop axes are all aligned in the $y$ direction, and the incident wave is propagating in the positive $z$ direction as in Fig. 6. The polarization state of the incident wave is either $x$-polarized (horizontal) or $y$-polarized (vertical) linear polarization. Because the scattering amplitude now depends on polarization, we may define two propagation constants in a rain-filled medium, $k_{x}$ and $k_{y}$ : 


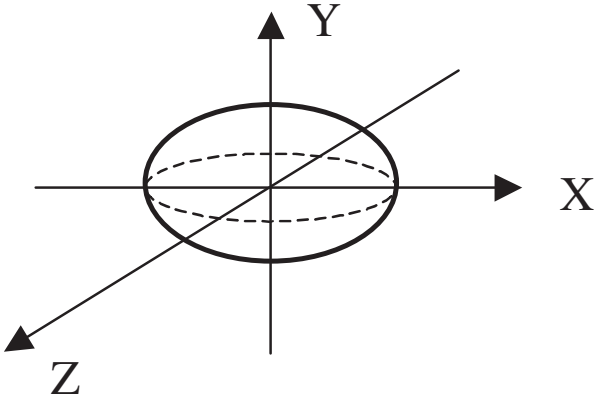

Fig. 6. Geometry of scattering from a spheroidal drop.

$$
\begin{aligned}
& k_{x} \\
& k_{y}
\end{aligned}=k_{0}+\frac{2 \pi}{k_{0}} \int \begin{gathered}
\hat{\mathbf{x}} \cdot \mathbf{f}_{x}\left(\hat{\mathbf{K}}_{1}, \hat{\mathbf{K}}_{1}, a\right) \\
\hat{\mathbf{y}} \cdot \mathbf{f}_{y}\left(\hat{\mathbf{K}}_{1}, \hat{\mathbf{K}}_{1}, a\right)
\end{gathered} n(a) d a
$$

where $a$ is the equivolume radius of a distorted drop.

From Eq. [6], attenuation difference $\Delta A$ (in $\mathrm{dB}$ difference) and phase difference $\Delta \phi$ (in degrees) between horizontal and vertical polarization channels are given by

$$
\begin{aligned}
\Delta A & =8.686 \times \operatorname{Im}\left(k_{x}-k_{y}\right) L \\
\Delta \phi & =(180 / \pi) \times \operatorname{Re}\left(k_{x}-k_{y}\right) L
\end{aligned}
$$

where $L$ is the propagation length (in meters) in a rain medium. These two quantities are usually termed "differential attenuation" for the former and "differential phase" for the latter. The theoretical curve, in Fig. 4, is the result of this perturbation calculation.

We then used the spheroidal function expansion method to obtain better results, since we expected spheroidal functions to have an affinity for spheroidal particles. The calculations should start from a detailed examination of the eigenvalues and the corresponding eigenfunctions of the spheroidal equation to define a complete set of eigenfunctions, because the eigenvalues have many branch points for complex values of propagation constants. ${ }^{25)}$ Unfortunately, the spheroidal elementary vector solutions lack orthogonal properties on the spheroidal boundary surface, and consequently, applying boundary conditions yields simultaneous linear equations of infinite extent to the unknown expansion coefficients of the fields to be determined. These equations were solved numerically after they were truncated at some modal index. ${ }^{11)}$ The results were naturally much better than those from perturbation calculation.

Computer technologies made rapid progress back then, and over time, computers were able to handle increasingly large amounts of matrix arith- metic. Our next solution was thus the collocation (point-matching) technique, or its improved version of matching in the sense of least squares. ${ }^{11)}$ In these techniques, incident, scattered, and transmitted fields are expanded by spherical elementary-vector solutions with known and unknown expansion coefficients. If the infinite modal summations are truncated at some modal index, and if the boundary conditions are satisfied for the representative points on the boundary, whose number is appropriate to the truncated modal index, these conditions yield simultaneous linear equations for determining the unknown coefficients. The procedure for solving simultaneous linear equations is almost analogous to that used in the spheroidal function expansion method, although the matrices are much larger. Note that this technique can flexibly be adapted to changes in the boundary shape. Thus, the scattering from Pruppacher-Pitter shaped raindrops was later calculated with the same technique.

A group at Bell Laboratories was conducting exactly the same research during almost the same period of time. Their research results were published just after our publication, as a series of papers in the Bell System Technical Journal. ${ }^{26)-28)}$ The results were compared by both sides with the same input data, and excellent agreement was confirmed.

The reasons a fairly large number of people at Bell Laboratories was involved in this research were as follows. Because of the ever-increasing demand for frequency resources, engineers in terrestrial and earth-space communication systems were planning to utilize frequency-reuse systems, such as those attained by employing two orthogonal polarizations at an identical frequency, to increase communications capacity. If coupling between two orthogonal polarization channels occurs, it will greatly limit the efficient use of these orthogonal channels. Distorted raindrops falling with trajectories canted from the direction of reference polarization will cause this interference, as will be described in the next section. This became the motivation for many experimental and theoretical investigations in the 1970s in various countries, such as the USA, many in Europe, and Japan.

Since then, many numerical techniques have been exploited, e.g., the T-matrix, ${ }^{29), 30)}$ the Fredholm integral-equation, ${ }^{31)}$ and the finite and boundaryelement methods. ${ }^{32), 33)}$ Recent comparisons of our past results with those obtained by the T-matrix and boundary-element methods have revealed that, at least for spheroidal and Pruppacher-Pitter raindrops 


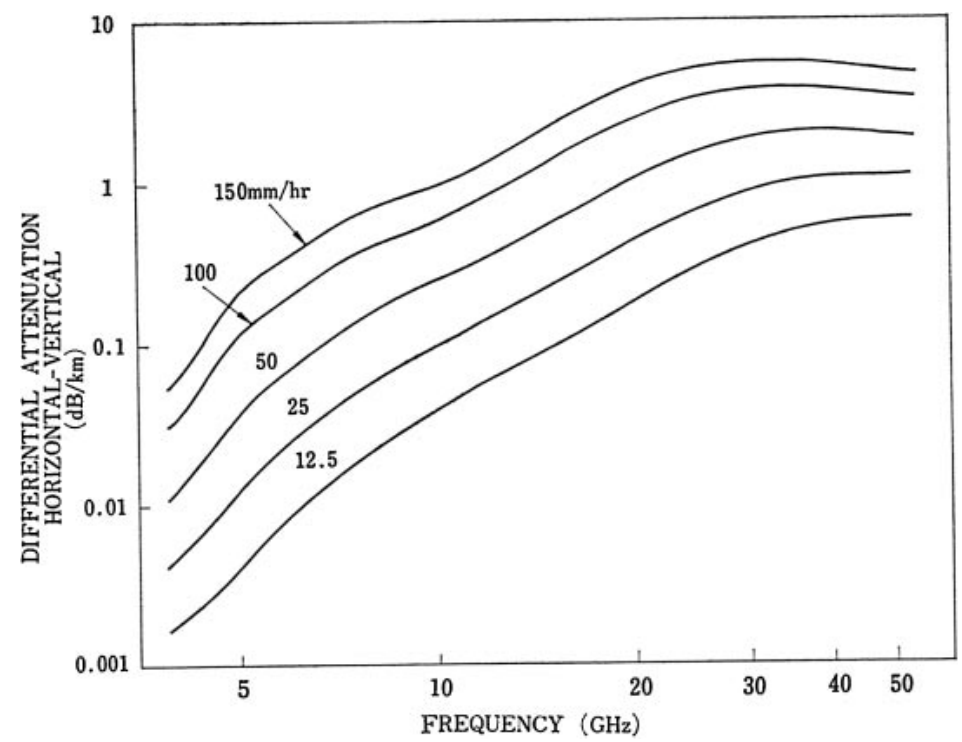

Fig. 7. Differential attenuation versus frequency. The parameter is rainfall rate. Drop axis is in vertical direction. (After Oguchi. ${ }^{10)}$ (C) 1983 IEEE.)

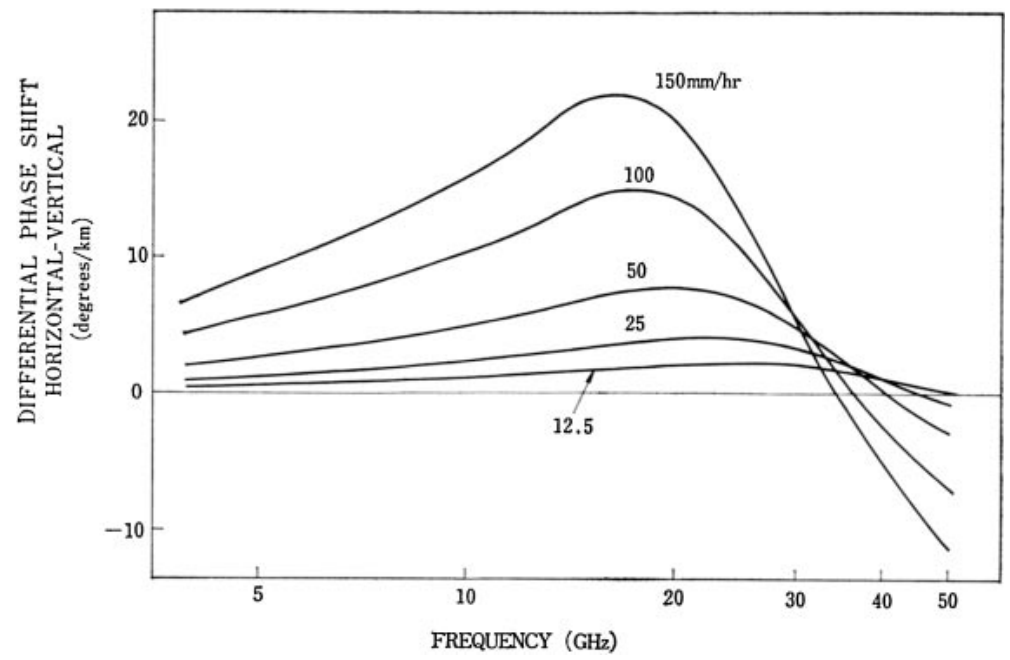

Fig. 8. Differential phase versus frequency. The parameter is rainfall rate. Drop axis is in vertical direction. (After Oguchi. ${ }^{10)}$ C) 1983 IEEE.)

in the microwave and millimeter-wave regions, agreement is excellent. ${ }^{34), 35)}$

4.3. Calculated polarization properties. Figures 7 and 8 plot the frequency characteristics of differential attenuation and differential phase defined by Eq. [7] for a 1-km propagation path. ${ }^{36)}$ The parameter is the rainfall rate. Spheroidal raindrops at a water temperature of $20^{\circ} \mathrm{C}$ and a Laws and Parsons drop-size distribution have been assumed. Figure 7 indicates that differential attenuation increases as frequency increases, and for high rainfall rates, it is maximum at about $30 \mathrm{GHz}$. Further increases in frequency result in a gradual decrease in differential attenuation. This is mainly due to the fact that, for frequencies higher than $30 \mathrm{GHz}$, the $\operatorname{Im}\left(f_{h}-f_{v}\right)$ of large drops decreases as frequency increases. It was fortunate that our attenuation measurements described in Section 3 were made at $34.8 \mathrm{GHz}$, the frequency that almost offers the maximum differential attenuation. Note from Fig. 8 that the differential phase is maximum at about $20 \mathrm{GHz}$, and this becomes negative for frequencies 


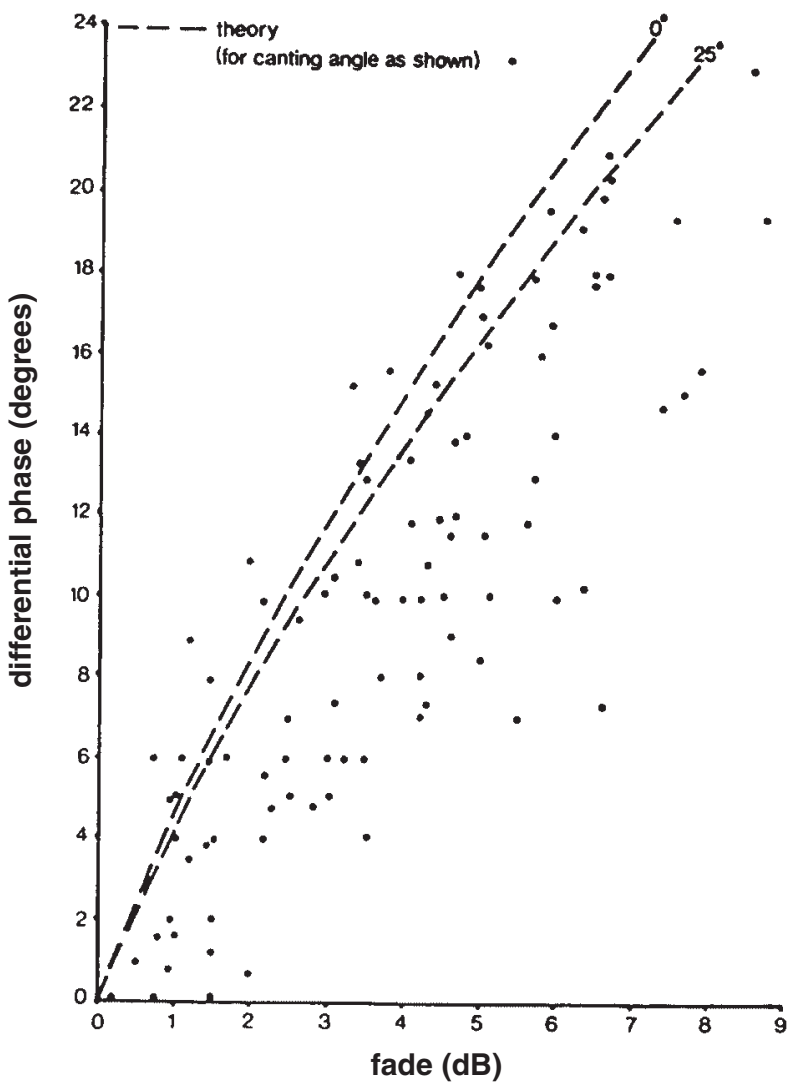

Fig. 9. Scatter plots of differential phase at $11 \mathrm{GHz}$ on a $13.6-\mathrm{km}$ propagation path as a function of attenuation in horizontal polarization. Dashed curves are the theoretical results for canting angles of $0^{\circ}$ and $25^{\circ}$. (After Watson and McEwan. ${ }^{37)}$ )

higher than $30 \mathrm{GHz}$. Negative differential phase shift can also be inferred from the single-scattering properties of nonspherical raindrops with their electrical size, $k_{0} a$, larger than one.

As shown in Fig. 4, the differential attenuation measured in 1960 agreed favorably with theoretical estimates. Because of the difficulty of doing phase measurements, we had to wait until the middle of the 1970s for measured data on differential phase shift to appear. Figure 9 shows an example of the differential phase measured at $11 \mathrm{GHz}$ on a $13.6-\mathrm{km}$ propagation path. ${ }^{37)}$ The theoretical curves are for drop canting angles of $0^{\circ}$ and $25^{\circ}$. The agreement between measured data and theoretical estimates is marginal. (The theoretical estimates based on a recent dropshape model, however, give better agreement with the measured data.) Although the differential attenuation and differential phase defined by Eq. [7] are the fundamental characteristics of a rain medium, they are in a sense for fictitious rainfall, because the

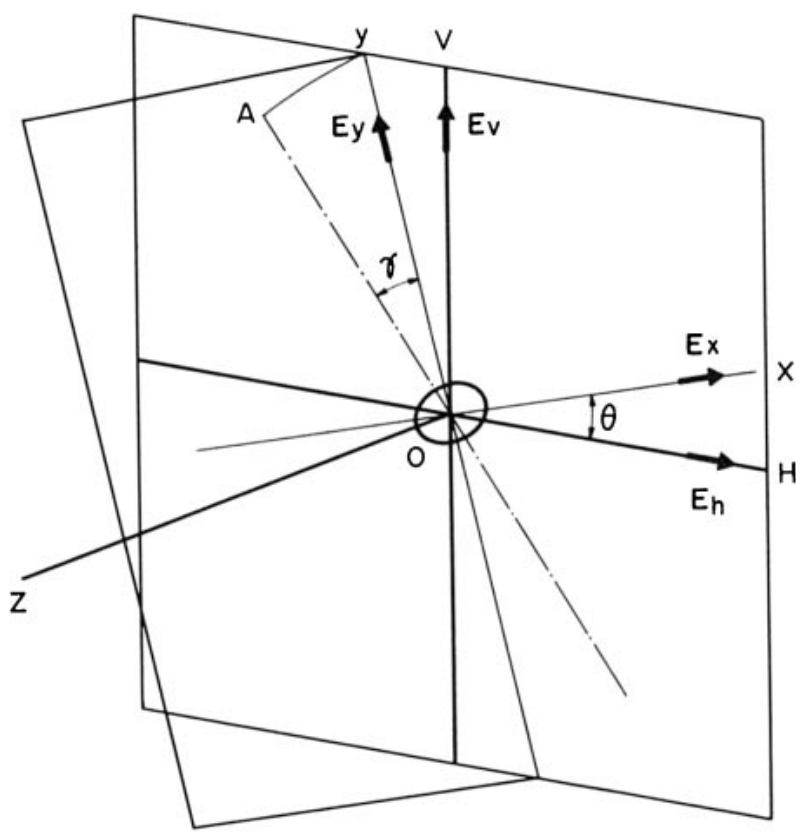

Fig. 10. Geometry for the calculation of cross-polarization factors for a canted raindrop. (After Oguchi. ${ }^{43)}$ (C) 1977 American Geophysical Union.)

raindrop axes are all vertically aligned. In real rain situations, however, drop axes are not necessarily vertical and they also have angle distributions. The procedure for treating more realistic rainfalls will be given in the next section.

\section{Effects of polarization on dual-polarization communication systems}

\subsection{Transmission equation of coherent wave} in rain. Many authors have reported on approximate or exact expressions giving equations describing the transmission of dual-polarized signals in rain. ${ }^{38)-43)}$ We here give our expressions since they are considered to be the most general. ${ }^{43)}$ It is assumed that the medium is statistically uniform, and the quantity of interest is the mean value of the field. Letting $H$ and $V$ be the two orthogonal directions of polarization for the transmitter and receiver, as shown in Fig. 10, a differential equation governing the variations in electric fields $E_{h}$ and $E_{v}$ of linear horizontal and vertical polarizations along propagation direction $z$ is written as

$$
d \mathbf{E} / d z=\mathbf{M E}
$$

where $\mathbf{E}$ is a column vector with elements $E_{h}$ and $E_{v}$, and $\mathrm{M}$ is a $2 \times 2$ square matrix. Matrix $\mathrm{M}$ is composed of two terms: 


$$
\mathrm{M}=\mathrm{M}_{0}+\mathrm{M}^{\prime}
$$

where $\mathrm{M}_{0}$, a diagonal matrix both of the elements being given by $-i k_{0}$, denotes the propagation characteristics of free space, and $\mathbf{M}^{\prime}$ denotes the effects of nonspherical raindrops with canting-angle distributions. We have considered a nonspherical raindrop whose symmetry axis $O A$ is in the $y z$ plane making angle $\gamma$ with axis $O y, O y$ being the projection of symmetry axis onto the $H V$ plane and inclined at angle $\theta$ from vertical axis $O V$ (see Fig. 10). The interaction of the incident wave with the drop can be expressed by the product of three matrices. The first matrix denotes the rotation of the direction of reference polarization from $(H, V)$ to $(x, y)$, the second matrix denotes the forward-scattering effects of the drop for $x$ - and $y$-polarized waves, and the third matrix denotes the back rotation of the direction of polarization from $(x, y)$ to $(H, V)$. (The second matrix is a diagonal matrix since forward scattering does not generate depolarized components for waves polarized in the $x$ and $y$ directions, i.e., the polarization directions that are normal to or in the plane containing the propagation direction and drop axis). The matrix, $\mathbf{M}^{\prime}$, is given by the sum of the matrices of all the raindrops in a unit volume with various sizes, deformation ratios, and axial directions.

The solution to Eq. [8] is written as

$$
\mathbf{E}=\mathrm{TE}^{0}
$$

where $\mathbf{E}^{0}$ is the electric field at $z=0$ and $\mathbf{T}$ denotes the transmission characteristics of the rain medium. The elements of $\mathrm{T}$ are given by

$$
\begin{aligned}
& T_{11}=\cos ^{2} \phi e^{\lambda_{2} z}\left(G+\tan ^{2} \phi\right) \\
& T_{12}=T_{21}=-\cos ^{2} \phi e^{\lambda_{2} z}(1-G) \tan \phi \\
& T_{22}=\cos ^{2} \phi e^{\lambda_{2} z}\left(1+G \tan ^{2} \phi\right)
\end{aligned}
$$

where

$$
\begin{aligned}
\phi & =\tan ^{-1} \frac{\kappa_{2}}{\kappa_{1}}=\frac{1}{2} \tan ^{-1} \frac{2 M_{12}}{M_{11}-M_{22}} \\
G & =\exp \left(\lambda_{1}-\lambda_{2}\right) z
\end{aligned}
$$

and where $\lambda_{1}$ and $\lambda_{2}$ are the eigenvalues of matrix $M$, and $\kappa_{1}$ and $\kappa_{2}$ are the elements of the corresponding eigenvectors. The angle, $\phi$, is generally complex. However, if the drop canting angle $(\theta, \gamma)$ is independent of the drop size and the two canting-angle distributions for $\theta$ and $\gamma$ are independent of each other, it can be shown that $\phi$ is real. (Several measurements have suggested that the above as- sumptions are approximately valid.) In particular, for the Gaussian distributions of canting angles with mean value $\theta_{0}$ and standard deviation $\sigma$ for $\theta$, and with mean value $\gamma_{0}$ and standard deviation $\sigma^{\prime}$ for $\gamma$, respectively, the difference in eigenvalues $\lambda_{1}-\lambda_{2}$ in Eq. [12] reduces to

$$
\lambda_{1}-\lambda_{2}=-i\left(k_{x}-k_{y}\right)(1 / 2)\left(e^{-2 \sigma^{\prime 2}} \cos 2 \gamma_{0}+1\right) e^{-2 \sigma^{2}}
$$

and it easily follows that $\phi=\theta_{0}$. The propagation constants, $k_{x}$ and $k_{y}$, are those defined by Eq. [6] in Section 4.

5.2. Cross-polarization factors. The degree of depolarization may be represented by the ratio of cross-polarized to copolarized signals at the receiver. From Eq. [10], cross-polarization isolations $X P I_{h}$ and $X P I_{v}$ for linear polarization are given by

$$
\begin{aligned}
X P I_{h} & =20 \log _{10}\left|\Delta E_{h} / E_{h}\right| \\
& =20 \log _{10}\left|T_{12} / T_{11}\right| \\
& =20 \log _{10}\left|(1-G) \tan \phi /\left(G+\tan ^{2} \phi\right)\right| \\
X P I_{v} & =20 \log _{10}\left|\Delta E_{v} / E_{v}\right| \\
& =20 \log _{10}\left|T_{21} / T_{22}\right| \\
& =20 \log _{10}\left|(1-G) \tan \phi /\left(1+G \tan ^{2} \phi\right)\right|
\end{aligned}
$$

where $X P I_{h}$ is the ratio of the cross-polarized received field $\Delta E_{h}$ in direction $E_{h}$ transferred from $E_{v}$ to copolarized received field $E_{h}$, and $X P I_{v}$ is a similar ratio when the direction of polarization of the wanted signal is vertical. Similar quantities often in use are cross-polarization discriminations (XPDs):

$$
\begin{aligned}
& X P D_{h}=20 \log _{10}\left|\Delta E_{v} / E_{h}\right| \\
& X P D_{v}=20 \log _{10}\left|\Delta E_{h} / E_{v}\right| .
\end{aligned}
$$

They are the ratios of cross-polarized received field in the orthogonal polarization channel to the copolarized received field. XPD occurs in the transmission measurements where a single-polarization transmitter and a dual-polarization receiver are used. If we take $E_{h}^{0}=E_{v}^{0}$ in Eq. [10] and use $T_{12}=T_{21}$, we find $X P D_{h}=X P I_{h}$ and $X P D_{v}=X P I_{v}$.

Transformation from linear to circular polarization is written as

$$
\mathbf{e}=\mathrm{CE}
$$

where $\mathbf{e}$ is a column vector whose two elements are right-hand and left-hand circular components of the electric field, and $\mathrm{C}$ is defined by

$$
\mathrm{C}=\frac{1}{\sqrt{2}}\left[\begin{array}{cc}
1 & i \\
1 & -i
\end{array}\right] .
$$

With the aid of Eq. [16], the transmission equa- 


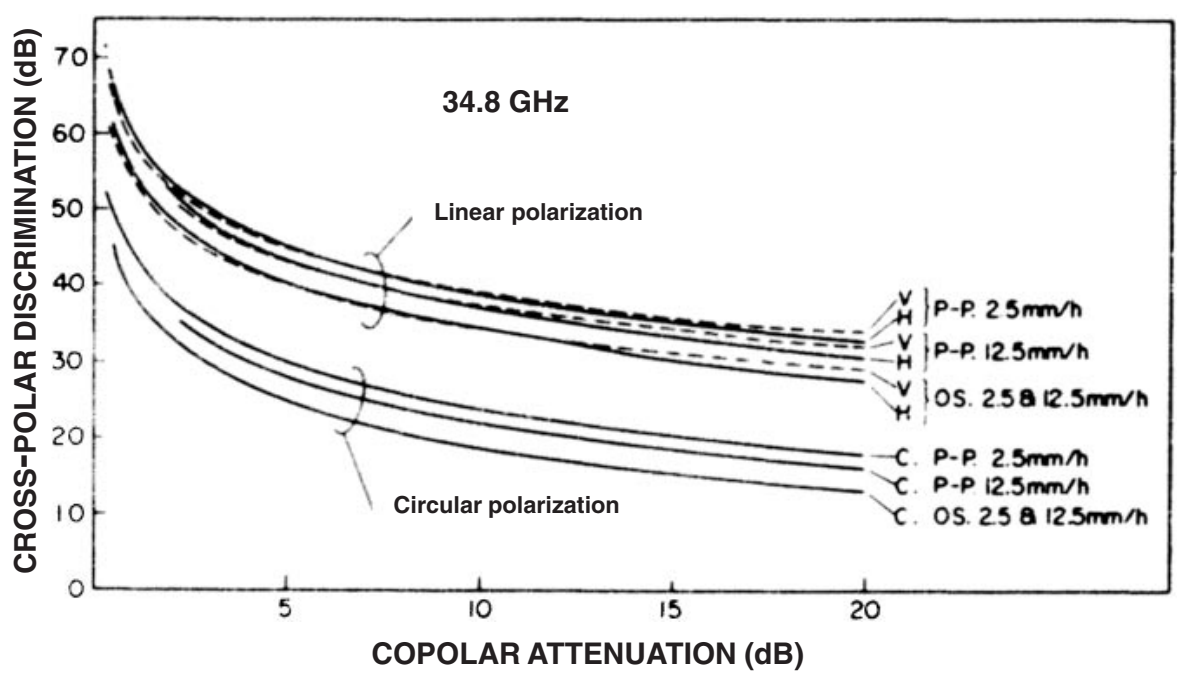

Fig. 11. Cross polarization versus copolar attenuation at $34.8 \mathrm{GHz}$ for equioriented oblate spheroidal and the Pruppacher-Pitter raindrops. Canting angles are $\theta_{0}=5^{\circ}$ and $\gamma=0^{\circ}$. In the figure key, $V, H$, and $C$ correspond to $X P D_{v}, X P D_{h}$, and $X P D_{r}\left(=X P D_{l}\right)$, and $\mathrm{P}-\mathrm{P}$ and $\mathrm{OS}$ correspond to the Pruppacher-Pitter and oblate spheroidal raindrops. (After Oguchi. ${ }^{10)}$ C) 1983 IEEE.)

tion [10] can be transformed into the corresponding equation for circular polarization

$$
\mathbf{e}=\mathrm{CTC}^{-1} \mathbf{e}^{0}
$$

where $\mathbf{e}^{0}$ is the value of $\mathbf{e}$ at $\mathbf{z}=0$. From Eq. [18], the cross-polarization factors for circular polarization are found to be

$$
\begin{aligned}
& X P I_{r}=20 \log _{10}\left|(1-G) e^{2 \phi i} /(1+G)\right| \\
& X P I_{l}=20 \log _{10}\left|(1-G) e^{-2 \phi i} /(1+G)\right|
\end{aligned}
$$

where $X P I_{r}$ and $X P I_{l}$ correspond to the cross-polarization factors when the polarizations of the wanted signal are right-hand and left-hand circular components and

$$
\begin{aligned}
& X P D_{r}=X P I_{l} \\
& X P D_{l}=X P I_{r} .
\end{aligned}
$$

If angle $\phi$ is real, $X P I_{r}=X P I_{l}$. Note from Eqs. [14] and [19] that, provided $\phi$ is real, the XPDs for circular polarization are the same as those for linear polarization for $\phi=45^{\circ}$, where $X P D_{h}\left(X P I_{h}\right)=X P D_{v}$ $\left(X P I_{v}\right)$ and has nearly the worst linear XPD values.

Figure 11 plots example calculated XPD values as a function of copolar attenuation (CPA) at 34.8 GHz. Equioriented spheroidal and PruppacherPitter raindrops have been considered $\left(\theta_{0}=5^{\circ}\right.$ and $\left.\gamma=0^{\circ}\right)$. The rainfall rates are 2.5 and $12.5 \mathrm{~mm} / \mathrm{h}$. It is evident that the XPDs for circular polarization are worse than those for linear polarization. The difference is about $15 \mathrm{~dB}$ in this example. Dual-polarization communication systems demand that the crosstalk resulting from depolarization should be better than $20 \mathrm{~dB}$ to ensure communications quality. In circular polarization, a copolarized signal attenuation of only 10 to $15 \mathrm{~dB}$ corresponds to this level of cross talk.

Several investigators have sought a simplified expression to describe the XPD-CPA relation, by applying small-argument approximation to the above expressions and by making further approximations. ${ }^{44-47)}$ A model XPD-CPA relation, thus devised for estimating depolarization, appeared in a report of the International Radio Consultative Committee (CCIR). ${ }^{48)}$

Figure 12 has an example comparison between measured scatter plots of XPD and calculations. The frequency is $34.5 \mathrm{GHz}$, and the polarization is circular. ${ }^{49)}$ The measurements were made along a $1.3-\mathrm{km}$ propagation path during a one-year period starting from April 1979. The Pruppacher-Pitter drop model, and $\sigma=0^{\circ}$ and $30^{\circ}$ with $\sigma^{\prime}=0^{\circ}$ in Eq. [13], were used in the calculations. It is evident from this figure that the measured plots are well within the two curves with $\sigma=0^{\circ}$ and $30^{\circ}$. Note that, in circular polarization, the XPDs are independent of the mean canting angle, $\phi$.

5.3. Adaptive depolarization compensation. In the above subsections, we discussed the mechanism for and the quantitative evaluation of rain depolarization. It was a natural consequence that investigations into techniques of compensating for depolarization coupling started at the same time. 


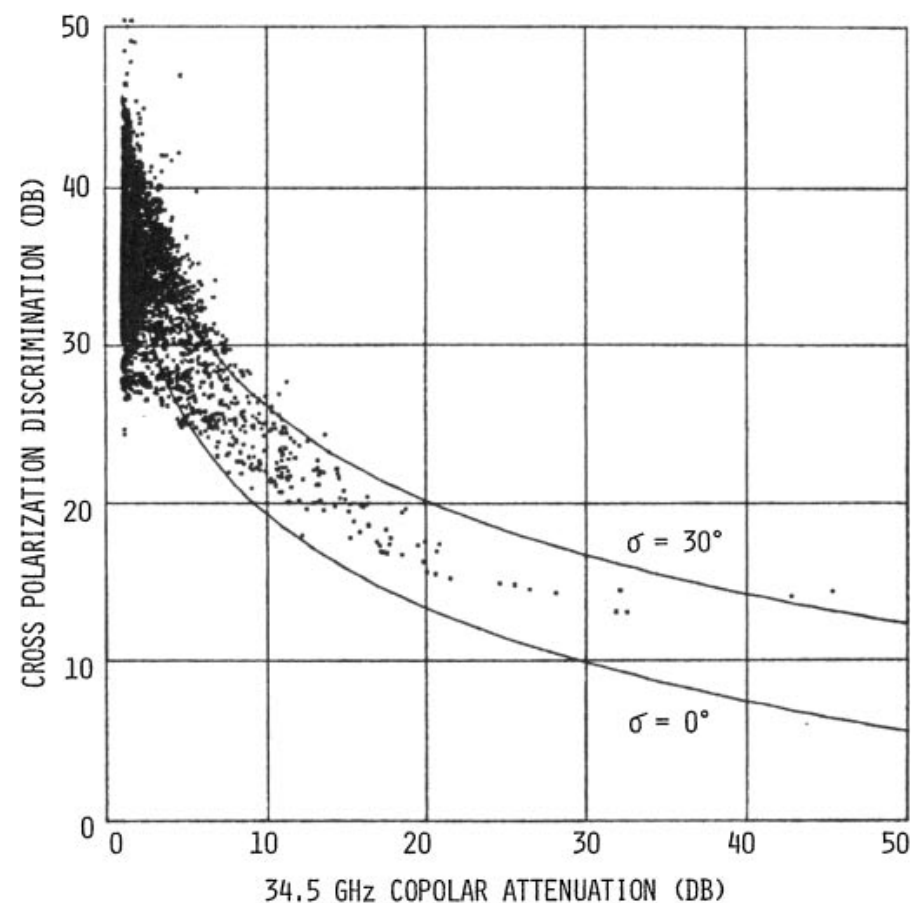

Fig. 12. Comparison of measured scatter plots of XPD with calculations at $34.5 \mathrm{GHz}{ }^{49)}$ Polarization is circular. Solid curves are the calculated results for the Pruppacher-Pitter drop model with canting angles of $\sigma=0^{\circ}$ and $30^{\circ}$, and $\sigma^{\prime}=0^{\circ}$.

Depolarization compensation is especially useful in earth-space communication systems, since the cost per channel is high. Because rain depolarization varies with time, compensation should be made on an adaptive basis.

Depolarization compensation is equivalent to finding the inverse of the transmission matrix. The compensation circuit may be placed in any stage of the receiver, i.e., $\mathrm{RF}$, IF, or baseband stages. Many scientists have proposed various techniques ${ }^{50)-62)}$ and several experimental investigations have been done. ${ }^{56), 57), 60), 62)}$ In the 4- and 6-GHz bands, differential attenuation is very small compared with the differential phase (see Fig. 8). Therefore, a combination of two rotatable differential phase shifters with fixed phase shift in the RF port can generate the inverse of the transmission matrix. An example of satellite propagation measurements with a circularly polarized signal in the $4-\mathrm{GHz}$ band indicated that, for depolarization levels of less than $15 \mathrm{~dB}$ lasting for more than $15 \mathrm{~min}$., the average value of the corrected levels was better than $30 \mathrm{~dB} .^{56)}$

Depolarization compensation is still being used. For instance, the newest antenna of the Yamaguchi Satellite Earth Station of KDDI Corporation, Japan, is equipped with a compensation system.

\section{Polarization effects in radar meteorology}

Radars are very important tools in meteorology for remotely monitoring the intensity of rain, as well as distinguishing solid hydrometeors (such as snow, or hail) from liquid (rain).

Meteorological radars were initially operated on the basis of single polarization. Conventional radars measure the backscattered intensity from hydrometeors in a scattering volume defined by the antenna-beam width and the transmittedpulse width. For a while, we will concentrate our discussion on rain with spherical raindrops. The received power, $P_{r}$, of a weather radar is written as

$$
P_{r}=\frac{C\left|K_{w}\right|^{2}}{r^{2}} Z
$$

where $C$ contains those parameters pertaining to the radar, such as transmitting power, antenna gain, antenna beamwidth, pulse width, etc., $r$ is the distance from the radar to the scattering volume, and $\left|K_{w}\right|^{2}=|(\varepsilon-1) /(\varepsilon+2)|^{2}$ is the dielectric factor of water with relative permittivity $\varepsilon$. Here, $Z$ is the reflectivity factor defined by the following equation customarily used in units of $\mathrm{mm}^{6} / \mathrm{m}^{3}$ 


$$
Z=\frac{\lambda^{4}}{\pi^{5}\left|K_{w}\right|^{2}} \int \sigma_{b} n(a) d a
$$

where $\lambda$ is the wavelength in free space (in $\mathrm{mm}$ ), $\sigma_{b}$ (in $\mathrm{mm}^{2}$ ) is the backscattering cross section of a raindrop with radius $a$, and $n(a)$ is the drop-size distribution function roughly approximated by the two-parameter negative exponential distribution, $\left.{ }^{15}\right)$ or, more strictly, by the three-parameter gamma distribution. ${ }^{63)}$ The following relation relates the backscattering cross section, $\sigma_{b}$, to the scattering amplitude in the backward direction, $\mathbf{f}$, if $\sigma_{b}$ is expressed in units of $\mathrm{m}^{2}$

$$
\sigma_{b}=4 \pi\left|\mathbf{f}\left(\hat{\mathbf{K}}_{1},-\hat{\mathbf{K}}_{1}\right)\right|^{2} .
$$

(Radar meteorologists use the term "equivalent reflectivity factor" for drops with sizes comparable to or larger than the wavelength (Mie-scattering region) to distinguish it from the commonly used "reflectivity factor" for drops much smaller than the wavelength (Rayleigh-scattering region). However, we will not distinguish these two cases and use the term "reflectivity factor". Although radar meteorologists prefer to use diameter $D$ to express the size of raindrops, we will use radius $a$ to maintain uniformity throughout this paper.)

The problem is whether we could find any definite relation between the radar observable, $Z$, and the rainfall rate, $R$, given by Eq. [5]. Although many radar meteorologists in the past have theoretically and experimentally studied the $Z-R$ relation, it has been difficult to find a definite relation for this. Measurements of the drop-size distribution function, $n(a)$, in rain have demonstrated that, even in one rain event, several different drop-size distributions have often yielded the same rainfall rate. Because variations in the drop-size distribution affect $Z$ differently, the $Z-R$ relation remains indefinite.

The polarization properties of scattering were then presented as a candidate for new radar observables whereby we could estimate $R$ more accurately. A dual-polarized radar transmits horizontally and vertically polarized pulses sequentially, and measures the reflectivity factors, $Z_{h}$ and $Z_{v}$, for these two polarizations. $Z_{h}$ and $Z_{v}$ are simply the extension of $Z$, with $\sigma_{b}$ in Eq. [22] replaced by those for horizontal and vertical polarizations. Assuming an a priori size-to-deformation relation of raindrops, and a near horizontal radar beam, Seliga and Bringi ${ }^{64), 65)}$ proposed to measure the differential reflectivity factor defined by $Z_{d r}=10 \log \left(Z_{h} / Z_{v}\right) . Z_{d r}$ and one of the absolute reflectivity factors, $Z_{h}$ or $Z_{v}$, can theoretically be used to define the two parameters of the exponential drop-size distribution. They also proposed using the differential phase, $\phi_{d p}$, between two polarization channels, in addition to $Z_{d r}$.

A distinctive feature of using the differential phase, $\phi_{d p}$, was demonstrated by Sachidananda and Zrnić. ${ }^{66)}$ The proposed algorithm, or its extension, is significant in accurate measurements of the rainfall rate, since it almost gives a direct relation between $\phi_{d p}$ and $R$ less dependent on the form of $n(a)$ as described below. The differential phase shift accumulates as the radar pulse penetrates into the depths of the rain-filled medium. Because rain is not necessarily uniform, the mean differential phase between the two adjacent ranges, $r_{i}$ and $r_{j}$, commonly termed specific differential phase $K_{d p}$, is given by the finite difference form of $\phi_{d p}$

$$
K_{d p}=\frac{\phi_{d p}\left(r_{i}\right)-\phi_{d p}\left(r_{j}\right)}{2\left(r_{i}-r_{j}\right)}
$$

$K_{d p}$ is the differential phase per unit distance, and is a function of the rainfall rate, drop shape, mean canting angle, and the standard deviation of canting angles; hence, in the strict sense, it should be evaluated from the transmission matrix, $\mathrm{T}$, with elements given by Eq. [11]. However, because our interest only lies in the copolar phase of orthogonalpolarization responses, the differential phase is almost the same as that of equi-oriented raindrops with their minor axes vertically aligned. Under this approximation, $K_{d p}$ is given by $\Delta \phi$ in Eq. [7] with $L=1$ :

$$
\begin{aligned}
K_{d p}= & \frac{360}{k_{0}} \operatorname{Re} \int\left[\hat{\mathbf{h}} \cdot \mathbf{f}_{h}\left(\hat{\mathbf{K}}_{1}, \hat{\mathbf{K}}_{1}, a\right)\right. \\
& \left.-\hat{\mathbf{v}} \cdot \mathbf{f}_{v}\left(\hat{\mathbf{K}}_{1}, \hat{\mathbf{K}}_{1}, a\right)\right] n(a) d a
\end{aligned}
$$

where Eq. [6] is used, and $x$ and $y$ in Eqs. [6] and [7] are replaced by $h$ and $v$. Sachidananda and Zrnic ${ }^{66)}$ demonstrated that, under the Rayleigh approximation, the above integral has dependence on $a^{4.24}$, while the rainfall-rate integral (Eq. [5]) approximately has dependence on $a^{3.67}$. In contrast to the dependence of the differential phase on $a^{4.24}$, the reflectivity factor, $Z$ (Eq. [22]), has dependence on $a^{6}$. Because the dependence of $K_{d p}$ and $R$ integrals on size is relatively close, the $K_{d p}-R$ relation is less sensitive to variations in $n(a)$ than the $Z-R$ relation. Note that the differential phase shift also includes the phase shift on scattering, in addition to the phase shift along the propagation path. However, this effect is minor for frequencies of less than, say, $3 \mathrm{GHz}$. 

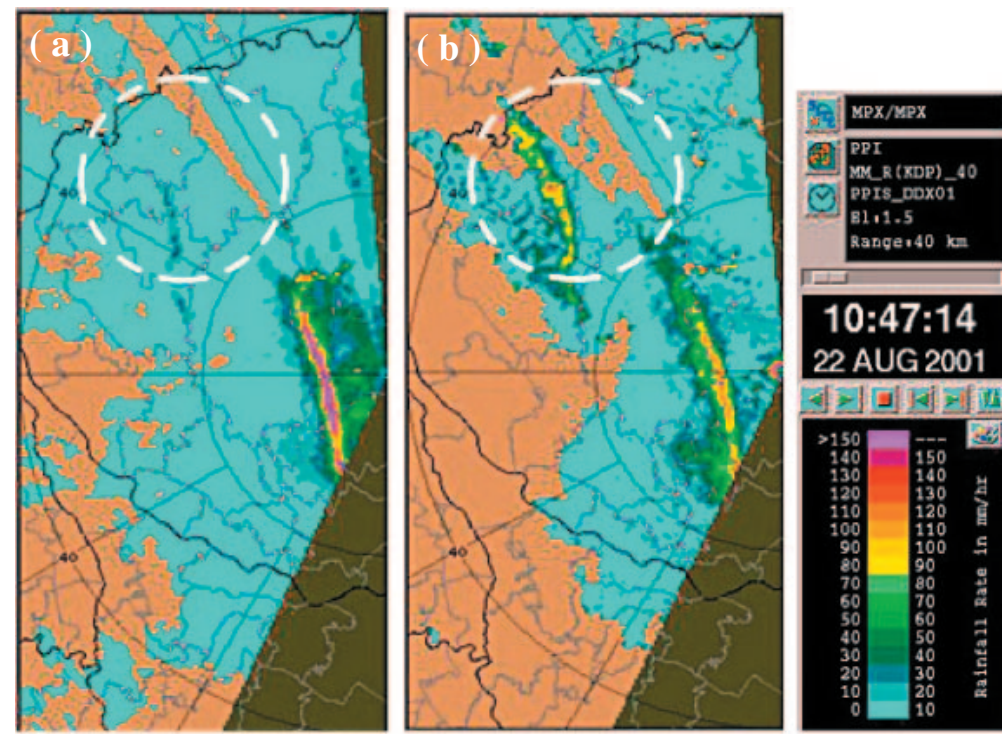

Fig. 13. Rainfall rate display of a typhoon (10:47:14 JST, 22 August 2001) measured by an X-band polarimetric radar: (a) Rainfall rate estimated by $Z-R$ relation and (b) rainfall rate estimated by $K_{d p}-R$ relation. (Courtesy M. Maki, Storm, Flood, and Landslide Research Department, National Research Institute for Earth Science and Disaster Prevention. ${ }^{70)}$ )

Investigations are still ongoing to find the best combinations of polarimetric radar observables. For instance, Maki et al. ${ }^{67)}$ found that further improvements in $R$ estimates might be possible if $R$ is estimated by using both $K_{d p}$ and $Z_{d r}$.

The polarimetric rainfall-rate estimator in operational use will be given, e.g., in the form $R=a\left(K_{d q}\right)^{b}$. Consequently, to find the most suitable numerical parameters, $a$ and $b$, it is fundamental to accurately know both the drop-size distribution functions and drop shapes that are representative of the radar-coverage area. Progress in these studies has recently been accelerated by the advent of new instruments, such as the two-dimensional video disdrometer, referred to in Section 4.1. ${ }^{21)-23)}$ The operational weather-forecast agencies in USA and Europe are planning to adopt dual-polarization capabilities for better forecasts of very localized and intense rainfall events. ${ }^{68)}$ In Japan, a polarimetric radar network for the Tokyo Metropolitan area is currently being investigated. ${ }^{69)}$

Figure 13 shows example rainfall-rate measurements obtained by using an X-band polarimetric radar under heavy rainfall associated with a typhoon. ${ }^{70)}$ While the rainfall rate in Fig. 13(a) was estimated with the conventional $Z-R$ relation, the rainfall rate in Fig. 13(b) was estimated with the $K_{d p}-R$ algorithm. The radar site is at the center of the right side of each panel. Although the area enclosed by the dashed circle in Fig. 13(a) does not reveal any noticeable rain activity, the same area in Fig. 13(b) clearly shows the existence of heavy rain. In Fig. 13(a), the reflection from the rain band in the circle seems to be masked by the attenuation of a strong rain band close to the radar site. In contrast to this, the $K_{d p}-R$ algorithm in Fig. 13(b) is less sensitive to the intervening rain attenuation between the radar and the rain cell being observed, since it uses phase information instead of using intensity information. This seems to be another benefit of using the $K_{d p}-R$ algorithm in addition to the already mentioned advantage of insensitivity to variations in drop-size distributions.

The use of polarization properties described thus far is not fully polarimetric, since it is lacking in information on cross-polar components. To derive full-polarimetric radar parameters, it is customary to define the two-by-two backscattering matrix, S, of a raindrop with the following equation, although it is equivalent to the vector-form scattering amplitude in the backward direction, $\mathbf{f}$ :

$$
\mathbf{E}^{b}=\mathbf{S E}^{i} r^{-1} \exp \left(-i k_{0} r\right)
$$

where $\mathbf{E}$ is a column vector with elements $E_{h}$ and $E_{v}$, and the superscripts $b$ and $i$ on $\mathbf{E}$ correspond to backscattered and incident waves. The radar observables are various second-order moments of the scattering matrix elements. In addition to $Z_{h}, Z_{v}$, and $Z_{d r}$ already defined, the other quantities often used in radar polarimetry are: ${ }^{71)}$ 
linear depolarization ratio (LDR)

$$
L D R_{h v}=10 \log \left(\left\langle\left|s_{h v}\right|^{2}\right\rangle /\left\langle\left|s_{v v}\right|^{2}\right\rangle\right)
$$

or

$$
L D R_{v h}=10 \log \left(\left\langle\left|s_{h v}\right|^{2}\right\rangle /\left\langle\left|s_{h h}\right|^{2}\right\rangle\right)
$$

copolar correlation coefficient

$$
\rho_{h v}=\left\langle s_{v v} s_{h h}^{*}\right\rangle /\left[\left\langle\left|s_{h h}\right|^{2}\right\rangle^{1 / 2}\left\langle\left|s_{v v}\right|^{2}\right\rangle^{1 / 2}\right]
$$

and cross-polar correlation coefficient

or

$$
\rho_{h}=\left\langle s_{h v} s_{h h}^{*}\right\rangle /\left[\left\langle\left|s_{h v}\right|^{2}\right\rangle^{1 / 2}\left\langle\left|s_{h h}\right|^{2}\right\rangle^{1 / 2}\right]
$$

$$
\rho_{v}=\left\langle s_{h v} s_{v v}^{*}\right\rangle /\left[\left\langle\left|s_{h v}\right|^{2}\right\rangle^{1 / 2}\left\langle\left|s_{v v}\right|^{2}\right\rangle^{1 / 2}\right]
$$

where $\langle\cdot\rangle$ denotes the averaging over drop sizes, shapes, canting angles, etc. Similar quantities for lefthand and right-hand circular-polarization bases can be defined.

LDR depends on the canting angle of distorted hydrometeors relative to the direction of reference polarization. The copolar correlation coefficient is generally close to one for rain. It has been pointed out both implicitly and explicitly that the cross-polar correlation coefficient, or its equivalent, might serve as a measure of the shape and orientation distributions of scattering particles ${ }^{72)-75)}$ Our laboratory measurements at Kanto Gakuin University in a simulated rain environment seem to support this statement. ${ }^{76)}$ Thus, using this coefficient may be helpful when using the depolarization ratio alone is insufficient to distinguish the effects of shape.

Radar meteorologists have made extensive studies up to the present on the microphysics of hydrometeors, i.e., not only raindrops but also ice crystals in high altitudes, snowflakes, hail, etc., by using full-polarimetric weather radars. We shall not go any deeper into this subject since it is beyond the scope of this paper. However, we do need to point out that McCormick and Hendry, and their colleagues have contributed much to the initial stages of research in this field. ${ }^{72)}$

\section{Multiple scattering effects}

7.1. Theoretical background. The discussion thus far has been based on the single-scattering regime. How multiple scattering of waves in rain affects attenuation and depolarization has been an unanswered question of long standing. This section briefly outlines our findings on the effects of multiple scattering. Unless otherwise stated, we have mainly considered spherical raindrops. The single scattering from spherical raindrops does not produce depolarized components, if the scattering occurs exactly forward or backward. However, when a wave successively travels through two or more scatterers, spherical raindrops can generate depolarized components even in exactly the forward or backward directions. The depolarization thus generated is often larger than that generated by non-spherical raindrops.

We used the vector radiative transfer equation as the basic expression for treating multiple scattering in rain with the polarization effects included. $\left.{ }^{77}\right)$ This equation can be derived from the more general Bethe-Salpeter type equation under ladder approximation. ${ }^{78), 79)}$ Although this is generally a good approximation, the so-called cyclical term is not included. The cyclical term is important in treating the enhanced backscattering that would occur if the transmitting and receiving antennas were in the same position. The problem of enhanced backscattering will be discussed in Section 7.3.

When we take into account the effects of polarization, we usually use the Stokes vectors that represent both intensities and the state of polarization. The radiative transfer equation for the Stokes vectors is an integro-differential equation, and the treatment of its integral is extremely difficult. We use circular polarization as a characteristic polarization state, since the representation of circular polarization yields simpler mathematics for the rotation of the coordinate axes in the integral than the representation of linear polarization does. ${ }^{80), 81)}$ Letting $E_{l}$ and $E_{r}$ be the electric-field components in left-hand and right-hand circular polarizations, the Stokes vector in the circular-polarization representation, $\mathbf{J}_{c p}$, is defined by $\mathbf{J}_{c p}=\left[\left\langle E_{l} E_{r}^{*}\right\rangle,\left\langle E_{l} E_{l}^{*}\right\rangle\right.$, $\left.\left\langle E_{r} E_{r}^{*}\right\rangle,\left\langle E_{r} E_{l}^{*}\right\rangle\right]^{T}$, where the asterisks denote complex conjugates, the brackets are for time averages, and superscript $T$ denotes the transpose. The Stokes vector, $\mathbf{J}_{c p}$, consists of the coherent part, $\mathbf{J}_{c p}^{0}$, and the incoherent part, $\mathbf{j}_{c p} . \mathbf{J}_{c p}^{0}$ corresponds to the singlescattering solution described in the previous sections. By expanding both $\mathbf{j}_{c p}$ and the kernel function in the integral in terms of generalized spherical functions, ${ }^{82)-84)}$ analytical integration in the equation of transfer involving Mie-scattering functions is feasible. This yields a set of simultaneous ordinary differential equations for the unknown functions to be determined, which is then to be numerically solved by using the matrix eigenvalue technique, or shooting technique, subject to proper boundary conditions. ${ }^{85), 86)}$ Further, if the expansion of $\mathbf{j}_{c p}$ is 
approximated by the first two terms, we have an approximate analytic solution. ${ }^{87), 88)}$ Although somewhat involved, the linear-polarization problem can similarly be solved by using the circular-polarization representation. ${ }^{86)}$

7.2. Monochromatic and pulse-wave propagation. First, we will summarize our findings on the co- and cross-polarized incoherent fields produced by multiple scattering in rain, when the incident wave is monochromatic. For communications purposes, our main concerns are coherent and incoherent fields exactly in the forward direction. Our calculations demonstrated that the incoherent effect is not an issue at frequencies ranging from the microwave to the low-frequency end of millimeter waves. The incoherent field in the copolarized channel is a noise component in a sense, and if the signal-to-noise ratio is assumed to be better than $20 \mathrm{~dB}$ as in Section 5.2, the copolarized incoherent component may become a problem at frequencies higher than $300 \mathrm{GHz}$ if a receiving antenna with $1^{\circ}$ half-power beamwidth is being used in heavy rainfall. ${ }^{89)}$ In the cross-polarized channel, the estimated incoherent intensity is an additional $10 \mathrm{~dB}$ below the level of copolarized incoherent intensity. Note that, in backward scattering, e.g., radar observation of rain, multiple scattering effects are often significant because of the lack of a coherent component, as will be discussed later.

The transmitted signals in communication systems and in remote sensing applications are commonly pulse modulated. Therefore, it is important to estimate the average incoherent pulse intensity and pulse shapes, with multiple scattering effects included, and to examine whether the multiple scattering in rain brings about unwanted effects in signal quality. To solve pulse problems, we use the time-dependent radiative transfer equation, or the two-frequency radiative transfer equation. Although the idea behind these two equations is different, it is common for these expressions to give the Fourier components of the Stokes vectors with respect to time, and the inverse Fourier transform then gives the incoherent wave-pulse shape as a function of time.

Numerical calculations of pulse shapes were made at $16,34.8$, and $140 \mathrm{GHz}$, for rainfall rates of 12.5 and $150 \mathrm{~mm} / \mathrm{h}^{90}$ ) The transmission of a Gaussian pulse with a half-power width of $0.5 \mu \mathrm{s}$ was assumed. The results indicate that, in forward scattering, the received power of the incoherent pulse generated by multiple-scattering effects is weak compared with that of the transmitted coherent pulses; thus, the incoherent pulses will not degrade the received signal quality. In backward scattering, multiple scattering will have relatively small effects on the interpretation of copolarized radar data. However, the calculated ratio of cross-polarized to copolarized incoherent intensities as a function of time indicates that, for a higher rainfall rate $(150 \mathrm{~mm} / \mathrm{h})$ or for higher frequencies (34.8 and $140 \mathrm{GHz}$ ), the effect of multiple scattering is stronger than that of drop distortion estimated by the firstorder calculations for Pruppacher-Pitter-shaped distorted drops. The ratio of cross-polarized to copolarized incoherent intensities, the LDR defined in Eq. [27] for linear polarization or a similar circulardepolarization ratio (CDR) for circular polarization, is an important parameter in radar polarimetry in idetifying the type of hydrometeors or in estimating the canting angle of distorted hydrometeors. The estimates of multiple scattering given thus far are based on theoretical investigations and are not necessarily supported by measurements. The following radar depolarization signatures of rain, obtained during air-borne radar measurements, would be a rare example demonstrating the importance of incoherent scattering.

In 1991, thunderstorm structures were measured with a $10 / 34.5 \mathrm{GHz}$ airborne radar in a joint experiment done by NASA and the Communictions Research Laboratory of Japan in Florida, USA. ${ }^{91)}$ The radar had two linear polarization channels for measuring both co- and cross-polarized returns in the $\mathrm{X}(10 \mathrm{GHz})$ and $\mathrm{Ka}$ bands $(34.5 \mathrm{GHz})$. The antenna beamwidths in the $\mathrm{X}$ and Ka bands were $5.2^{\circ}$ for the former and $5.1^{\circ}$ for the latter, and the pulse width was $0.5 \mu \mathrm{s}$ for both frequency bands. The radar beam was directed close to the nadir. Figure 14 compares the measured and calculated LDR values for both $\mathrm{X}$ and Ka bands. ${ }^{92)}$ The rain had a stratified structure of thickness of about $3 \mathrm{~km}$ downwards, starting from about $3.5 \mathrm{~km}$ below the aircraft. The rainfall rate estimated from the measured reflectivity was $25 \mathrm{~mm} / \mathrm{h}$, and this value was used in the calculations. It is evident from Fig. 14 that the LDR gradually increases as the radar pulse penetrates into the rain region. In the $\mathrm{Ka}$ band, the agreement between measured and calculated values is satisfactory up to the range of about $5.5 \mathrm{~km}$ from the aircraft. Further increases in range result in poor agreement, possibly because the very weak radar returns caused by the intervening rain attenuation between the radar and the rain cell are responsible for the unreliable LDR values. (The $\mathrm{LDR}$ value of $-25 \mathrm{~dB}$ in the $\mathrm{X}$ band 


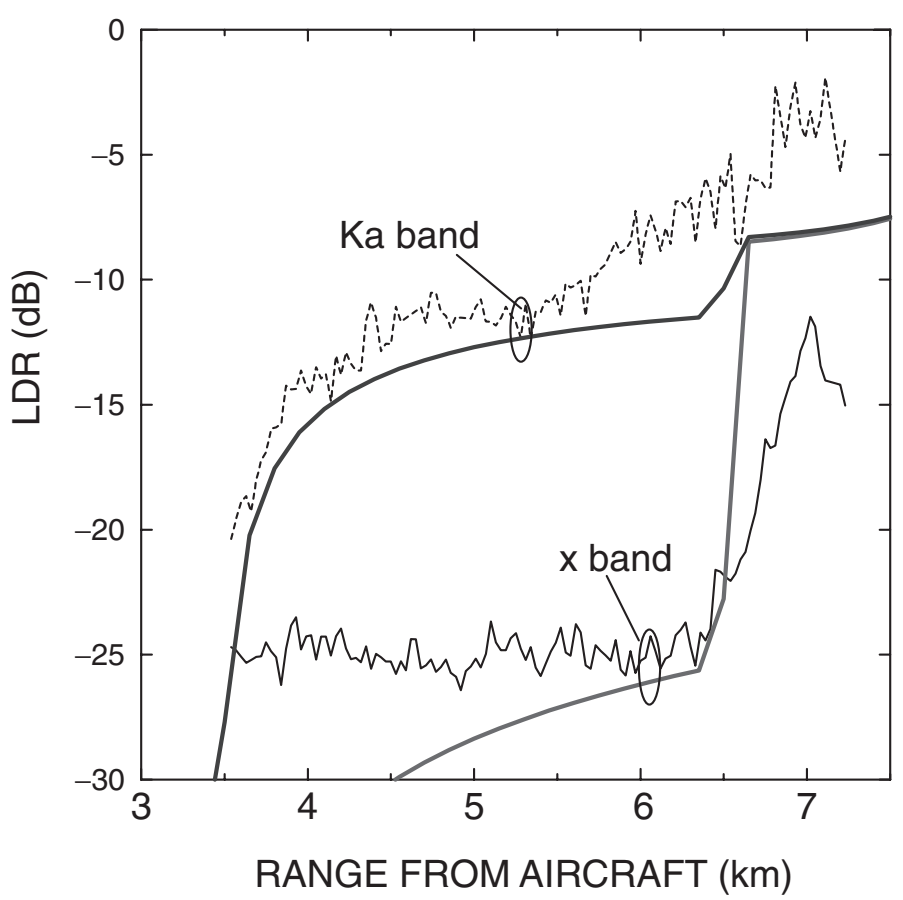

Fig. 14. Comparison of measured and calculated LDR values for $\mathrm{X}$ and Ka bands. The airborne radars were pointing close to the nadir, and the rain had a stratified structure of thickness of about $3 \mathrm{~km}$ downwards starting from about $3.5 \mathrm{~km}$ below the aircraft. Two thick solid curves are the calculated results by the second-order solution to the time-dependent radiative transfer equation. Estimated rainfall rate of $25 \mathrm{~mm} / \mathrm{h}$ was used in the calculation. (Reproduced from Figs. 3 and 4 of Ito et al. ${ }^{92)}$ (C) 1995 IEEE.)

seems to be the minimum detectable level determined by the polarization isolation of the X-band antenna.) The Ka-band's LDR value reaches as high as $-10 \mathrm{~dB}$, and this value cannot be explained by depolarization based on distorted raindrops. It is interesting to note that the LDR values increase rapidly near the rear edge of the rain layer $(\sim 6.5 \mathrm{~km}$ from the aircraft). This was caused by the fact that the major copolarized component (first-order scattering) vanished beyond the rear edge, whereas multiple-scattered components survived even in that time region due to their long electrical paths and they caused large LDR values. This is a very clear indication of the existence of multiple scattering.

In the pulse-propagation problems discussed thus far, we have assumed the transmission of a plane wave pulse. Actual radars, however, transmit pulsed beam waves corresponding to their antenna beamwidths. The multiple scattering theory of pulse-wave propagation has very recently been extended to account for pulsed beam waves. ${ }^{93), 94)}$ Numerical calculations at $95 \mathrm{GHz}$ for several antenna beamwidths indicate that the strengths of the incoherent components decreases as the beamwidth narrows. ${ }^{94)}$

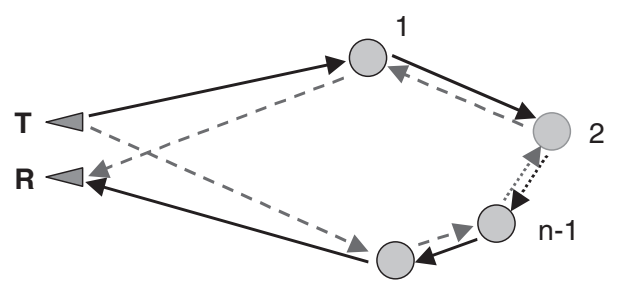

Fig. 15. Schematic diagram of multiple scattering process.

7.3. Backscattering enhancement. The theoretical treatments given above only include the contribution of a ladder term without regard for the contribution of the so-called cyclical term known in diagrammatical theory. The contribution of the cyclical term is often important when radars are operating with a monostatic configuration. When a transmitted wave travels through $n$ successive scattering centers $(n \geq 2)$ in a scattering medium from scatterer 1 to scatterer $n$ and enters a receiver as outlined in Fig. 15, there is also another wave in the medium that travels in the opposite direction from scatterer $n$ to scatterer 1 (time-reversed path) exactly on the same channel, as indicated by the dashed lines. 
If the transmitting and receiving antennas are in the same position, these two waves will be added in phase resulting in enhanced backscattering. Therefore, enhancement is a peculiar phenomenon that occurs when the scattering angle is very narrow. (The scattering angle is the angle of intersection of two antenna beams for the transmitter and receiver on the front face of the scattering volume being investigated.) It is suggested that the angular range of enhancement is of the order of a wavelength divided by the mean free path of scattering; the mean free path being given by the reciprocal of the total cross section per unit volume. A typical angular range of enhancement in radar meteorology is less than $1^{\circ}$. The enhanced backscattering is a well-known phenomenon in various branches of physics, such as in optics and condensed matter physics. ${ }^{95)}$ In the theoretical treatment of enhancement in electromagnetic-wave scattering from random discrete scatterers, second-order theory in conjunction with plane-wave incidence has commonly been used. ${ }^{96), 97)}$ The theory has very recently been extended to the case of finite beam width, ${ }^{98)}$ as well as to spheroidshaped hydrometeors. ${ }^{34)}$ The effect of multiple scattering including enhanced backscattering is increasingly important as the radar frequency increases. In fact, the use of the high-frequency region of millimeter waves, such as $94 \mathrm{GHz}$ used in the spaceborne radar on board the NASA CloudSat satellite, has aroused scientific interest in multiple scattering. It must be noted, however, that enhanced backscattering is not necessarily expected in spaceborne radars, because when the radar has received scattered signals, the radar platform itself is displaced from the transmitting position, hence creating an apparent scattering angle between the transmitting and receiving antennas. For ground-based monostatic weather radars operating in this frequency range, on the other hand, the existence of enhanced backscattering would not be ignored depending on the meteorological conditions.

Finally, we will briefly present the results of laboratory measurements and numerical simulations of enhanced backscattering. ${ }^{99), 100)}$ Laboratory measurements of enhanced backscattering from randomly distributed large water scatterers were done at $30 \mathrm{GHz}$ under a controlled scattering environment. The computer simulation model was constructed to adhere to exactly the same scattering environment used in the experiment. We used 1736 water-injected thin polystyrene spheres as the scatterers with a radius of $12.5 \mathrm{~mm}$ randomly positioned in a scatter-
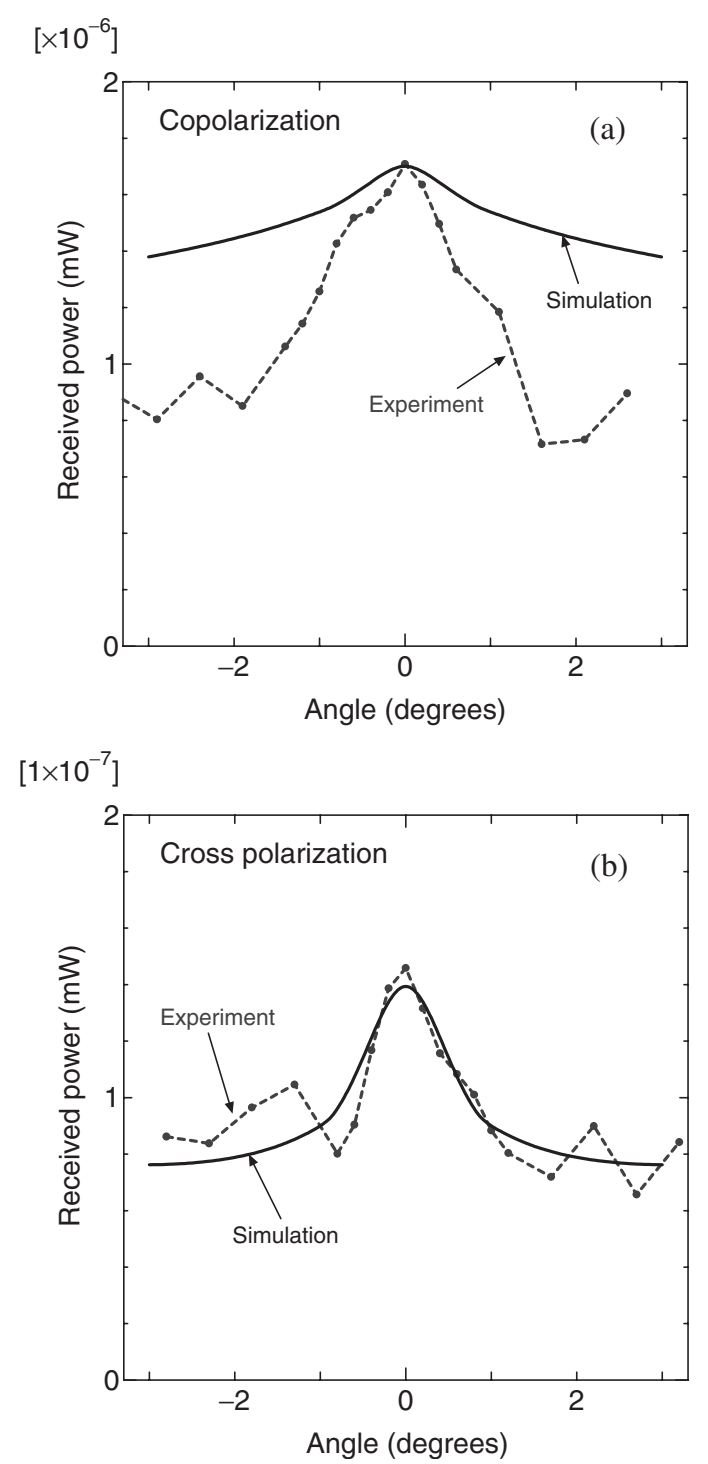

Fig. 16. Comparison of simulated received powers with measurement: (a) Copolarized and (b) cross-polarized received powers. Incident-wave polarization is vertical. (After Oguchi and Ihara. ${ }^{100)}$ (C) 2006 American Geophysical Union.)

ing volume of $0.9 \times 0.9 \times 0.9 \mathrm{~m}$. The distance from the transmitter to the front face of the scattering volume was $3.15 \mathrm{~m}$. Figure 16 plots the measured and simulated received powers as a function of the scattering angle. The simulated copolarized values contain the contribution of single scattering in addition to the multiple-scattered (ladder plus cyclical) contribution, while the cross-polarized values only contain the multiple-scattered contribution. The simulation results agree favorably with those of the measurements in cross-polarized values. 
In the copolarized channel, however, the agreement is poor. (A reasonable explanation for this has not been given as yet. Further study is therefore needed to reexamine the results.) The angular range of enhancement estimated by using the parameters of the scattering environment was approximately $0.8^{\circ}$, which is in fairly good agreement with the measured and simulated curves. Although the scattering environment was not an actual rain environment constructed to scale, nevertheless, the results contain many important features of enhanced backscattering that would occur in real rain situations.

\section{Conclusions}

We surveyed the progress of research related to the effects of polarization on electromagnetic wave propagation and scattering in rain in this paper. Half a century has passed since our finding that rain attenuation in horizontal polarization is slightly larger than that in vertical polarization. Although such a small polarization effect was initially considered to not have any practical importance, the effects of polarization were later found to be very important factors related to radiowave communications in rain and the monitoring of rain by meteorological radars.

The depolarization phenomena caused by distorted raindrops were intensively studied in various countries in the 1970s, in connection with dualpolarization communication systems. Investigations into techniques for compensating for depolarization coupling were also conducted. In the 4 - and $6-\mathrm{GHz}$ bands, depolarization compensation is feasible only due to the compensation of differential phase, because attenuation itself is very small. For instance, the newest antenna at the Yamaguchi Satellite Earth Station of KDDI Corporation, Japan, is equipped with a compensation system.

The differential phase has recently again been spotlighted in polarimetric radar meteorology, since it is a very effective estimator of the rainfall rate. It is interesting to note that in Japan, a polarimetric radar network for the Tokyo Metropolitan area is being investigated so that it can provide better forecasts of very localized and intense rainfall events.

It is thus fortunate that our past findings on polarization-dependent rain attenuation have been closely related to, or have initiated in some cases, future progress in polarimetric studies on rain.

\section{Acknowledgements}

The authors would like to thank Dr. K. Funakawa (formerly with the Radio Research Laboratory, and later with the National Space Development Agency of Japan), Dr. H. Uda (who had the same professional career as Dr. Funakawa, but who is now unfortunately deceased) and Mr. J. Kato, who are former members of the Millimeter Wave Research Section of the Radio Research Laboratory. Without their dedication, it would have been impossible for the rain-attenuation measurements to have been made. The authors would also like to thank Dr. K. Ono (KDDI Corporation) and Prof. T. Ihara (Kanto Gakuin University) for their invaluable pieces of information. Finally, the authors would like to thank the anonymous reviewers for their constructive comments in revising this paper.

\section{References}

1) Ryde, J.W. (1941) Echo Intensity and Attenuation due to Clouds, Rain, Hail, Sand and Duststorms at Centimetre Wavelengths. Rep. 7831, General Electric Co. Research Labs., Wembley, England.

2) Ryde, J.W. and Ryde, D. (1944) Attenuation of Centimetre Waves by Rain, Hail and Clouds. Rep. 8516, General Electric Co. Research Labs., Wembley, England.

3) Ryde, J.W. and Ryde, D. (1945) Attenuation of Centimetre and Millimetre Waves by Rain, Hail, Fogs and Clouds. Rep. 8670, General Electric Co. Research Labs., Wembley, England.

4) Robertson, S.D. and King, A.P. (1946) The effect of rain upon the propagation of waves in the 1- and 3-centimeter regions. Proc. Inst. Radio Eng. 34, 178-180.

5) Mueller, G.E. (1946) Propagation of 6-millimeter waves. Proc. Inst. Radio Eng. 34, 181-183.

6) Okamura, S., Funakawa, K., Uda, H., Kato, J. and Oguchi, T. (1961) Effect of polarization on the attenuation by rain at millimeter-wave length. J. Radio Res. Labs. (Japan) 8, 73-80.

7) Magono, C. (1954) On the shape of water drops falling in stagnant air. J. Meteorol. 11, 77-79.

8) Oguchi, T. (1960) Attenuation of electromagnetic wave due to rain with distorted raindrops. J. Radio Res. Labs. (Japan) 7, 467-485; Oguchi, T. (1988) Reproduced in "Selected papers on Light Scattering" (ed. Kerker, M.). SPIE Milestone Series, SPIE 951, 589-605.

9) Atlas, D., Kerker, M. and Hitschfeld, W. (1953) Scattering and attenuation by non-spherical atmospheric particles. J. Atmos. Terr. Phys. 3, 108-119.

10) Oguchi, T. (1983) Electromagnetic wave propagation and scattering in rain and other hydrometeors. Proc. IEEE 71, 1029-1078.

11) Oguchi, T. (1973) Attenuation and phase rotation of radio waves due to rain: Calculations at 19.3 and 34.8 GHz. Radio Sci. 8, 31-38.

12) Saxon, D.S. (1955) Tensor scattering matrix for the electromagnetic field. Phys. Rev. 100, 1771-1775. 
13) Stratton, J.A. (1941) Electromagnetic Theory. McGraw-Hill, New York.

14) Laws, J.O. and Parsons, D.A. (1943) The relation of raindrop-size to intensity. Trans. Am. Geophys. Union 24, 452-460.

15) Marshall, J.S. and Palmer, W.McK. (1948) The distribution of raindrops with size. J. Meteorol. 5, $165-166$

16) Rogers, D.V. and Olsen, R.L. (1976) Calculation of radiowave attenuation due to rain at frequencies up to $1000 \mathrm{GHz}$. Rep. 1299, Comm. Res. Cen., Dept. of Commun., Ottawa, Canada.

17) Okamura, S., Funakawa, K., Uda, H., Kato, J. and Oguchi, T. (1959) On the measurement of attenuation by rain at $8.6 \mathrm{~mm}$ wave length. J. Radio Res. Labs. (Japan) 6, 255-267.

18) Crawford, A.B. and Hogg, D.C. (1956) Measurement of atmospheric attenuation at millimeter wavelengths. Bell Syst. Tech. J. 35, 907-916.

19) Semplak, R.A. (1970) Effect of oblate raindrops on attenuation at $30.9 \mathrm{GHz}$. Radio Sci. 5, 559-564.

20) Pruppacher, H.R. and Pitter, R.L. (1971) A semiempirical determination of the shape of cloud and rain drops. J. Atmos. Sci. 28, 86-94.

21) Thurai, M. and Bringi, V.N. (2005) Drop axis ratios from 2D video disdrometer. J. Atmos. Ocean. Technol. 22, 966-978.

22) Thurai, M., Huang, G.J., Bringi, V.N., Randeu, W.L. and Schönhuber, M. (2007) Drop shapes, model comparisons, and calculations of polarimetric radar parameters in rain. J. Atmos. Ocean. Technol. 24, 1019-1032.

23) Thurai, M., Szakáll, M., Bringi, V.N., Beard, K.V., Mitra, S.K. and Borrmann, S. (2009) Drop shapes and axis ratio distributions: Comparison between $2 \mathrm{D}$ video disdrometer and wind-tunnel measurements. J. Atmos. Ocean. Technol. 26, 1427-1432.

24) Mushiake, Y. (1956) Backscattering for arbitrary angles of incidence of a plane electromagnetic wave on a perfectly conducting spheroid with small eccentricity. J. Appl. Phys. 27, 1549-1556.

25) Oguchi, T. (1970) Eigenvalues of spheroidal wave functions and their branch points for complex values of propagation constants. Radio Sci. 5, $1207-1214$.

26) Morrison, J.A., Cross, M.-J. and Chu, T.S. (1973) Rain-induced differential attenuation and differential phase shift at microwave frequencies. Bell Syst. Tech. J. 52, 599-604.

27) Morrison, J.A. and Chu, T.S. (1973) Perturbation calculations of rain-induced differential attenuation and differential phase shift at microwave frequencies. Bell Syst. Tech. J. 52, 1907-1913.

28) Morrison, J.A. and Cross, M.-J. (1974) Scattering of a plane electromagnetic wave by axisymmetric raindrops. Bell Syst. Tech. J. 53, 955-1019.

29) Waterman, P.C. (1965) Matrix formulation of electromagnetic scattering. Proc. IEEE 53, 805812 .

30) Mishchenko, M.I. (2000) Calculation of the amplitude matrix for a nonspherical particle in a fixed orientation. Appl. Opt. 39, 1026-1031.
31) Holt, A.R., Uzunoglu, N.K. and Evans, B.G. (1978) An integral equation solution to the scattering of electromagnetic radiation by dielectric spheroids and ellipsoids. IEEE Trans. Antennas Propag. AP-26, 706-712.

32) Morgan, M.A. (1980) Finite element computation of microwave scattering by raindrops. Radio Sci. 15, $1109-1119$.

33) Paulsen, K.D., Lynch, D.R. and Strohbehn, J.W. (1988) Three-dimensional finite, boundary, and hybrid element solutions of the Maxwell equations for lossy dielectric media. IEEE Trans. Microw. Theory Tech. 36, 682-693.

34) Kobayashi, S., Oguchi, T., Tanelli, S. and Im, E. (2007) Backscattering enhancement on spheroidshaped hydrometeors: Considerations in water and ice particles of uniform size and MarshallPalmer distributed rains. Radio Sci. 42, RS2001, doi:10.1029/2006RS003503.

35) Duffo, N., Vall-llossera, M., Camps, A., Corbella, I. and Torres, F. (2009) Polarimetric emission of rain events: Simulation and experimental results at X-band. Remote Sens. 1, 107-121; doi:10.3390/ rs1020107.

36) Oguchi, T. and Hosoya, Y. (1974) Differential attenuation and differential phase shift of radio waves due to rain: Calculations at microwave and millimeter wave regions. J. Rech. Atmos. (Universités de Clermont et de Toulouse, France) 8, 121-128; Oguchi, T. and Hosoya, Y. (1974) Scattering properties of oblate raindrops and cross polarization of radio waves due to rain (Part II): Calculations at microwave and millimeter wave regions. J. Radio Res. Labs. (Japan) 21, 191-259.

37) Watson, P.A. and McEwan, N.J. (1974) Cross polarisation, attenuation and radar reflectivity studies at X-band, Final report submitted to the ESRO under contract 2044/73MD.

38) Attisani, A., Capsoni, C. and Paraboni, A. (1974) Effect of non-spherical hydrometeors on EM propagation through atmospheric precipitation. J. Rech. Atmos. (Universités de Clermont et de Toulouse, France) 8, 137-145.

39) Chu, T.S. (1974) Rain-induced cross-polarization at centimeter and millimeter wavelengths. Bell Syst. Tech. J. 53, 1557-1579.

40) McCormick, G.C. (1975) Propagation through a precipitation medium: Theory and measurement. IEEE Trans. Antennas Propag. AP-23, 266-269.

41) Brussaard, G. (1976) A meteorological model for rain-induced cross polarization. IEEE Trans. Antennas Propag. AP-24, 5-11.

42) Östberg, K. (1976) Effect of drop canting-angle distribution on depolarization of microwaves in rain. National Defence Research Institute, Stockholm, Sweden FOA Rep. 10(2).

43) Oguchi, T. (1977) Scattering properties of Pruppacher-and-Pitter form raindrops and cross polarization due to rain: Calculations at 11, 13, 19.3, and $34.8 \mathrm{GHz}$. Radio Sci. 12, 41-51.

44) Nowland, W.L., Olsen, R.L. and Shkarofsky, I.P. (1977) Theoretical relationship between rain 
depolarisation and attenuation. Electron. Lett. 13, 676-678.

45) Olsen, R.L. and Nowland, W.L. (1978) Semiempirical relations for the prediction of rain depolarization statistics: Their theoretical and experimental basis. Proc. Int. Symp. on Antennas and Propagation (Japan), 477-480.

46) Chu, T.S. (1982) A semi-empirical formula for microwave depolarization versus rain attenuation on earth-space paths. IEEE Trans. Commun. COM-30, 2550-2554.

47) Dissanayake, A.W., Haworth, D.P. and Watson, P.A. (1980) Analytical models for cross-polarization on earth-space radio paths for frequency range 9-30 GHz. Ann. Télécommunic. 35, 398404.

48) CCIR, Rep. 722-1 (1982) Cross-polarization due to the atmosphere. Recommendations and Reports of the CCIR. 5, 185-193 (Int. Telecommun. Union, Geneva, Switzerland).

49) Radio Wave Div. Rep. (1981) Feasibility study of using radio wave spectrum over $40 \mathrm{GHz}$. Pt. II. Radio Wave Div., Radio Res. Labs., Tokyo, Japan (Jan. 1981) (in Japanese).

50) Chu, T.S. (1971) Restoring the orthogonality of two polarizations in radio communication systems, I. Bell Syst. Tech. J. 50, 3063-3069.

51) Chu, T.S. (1973) Restoring the orthogonality of two polarizations in radio communication systems, II. Bell Syst. Tech. J. 52, 319-327.

52) Kreutel, R.W. (1973) The orthogonalization of polarized fields in dual-polarized radio transmission systems. COMSAT Tech. Rev. 3, 375386.

53) DiFonzo, D.F., Trachtman, W.S. and Williams, A.E. (1976) Adaptive polarization control for satellite frequency reuse systems. COMSAT Tech. Rev. 6, 253-283.

54) Williams, A.E. and Frey, F.L. (1976) Adaptive polarisation control on a dual-polarised $4 / 6 \mathrm{GHz}$ SATCOM link in the presence of rain. Electron. Lett. 12, 686-687.

55) Kannowade, H. (1976) An automatic control system for compensating cross-polarization coupling in frequency-reuse communication systems. IEEE Trans. Commun. COM-24, 986-999.

56) Williams, A.E. (1977) A dual-polarized $4 / 6 \mathrm{GHz}$ adaptive polarization control network. COMSAT Tech. Rev. 7, 247-262.

57) DeLogne, P. and Sobieski, P. (1978) Adaptive cancellation of crosspolarisation: Experiments with unmodulated signals. Electron. Lett. 14, 770-772.

58) Overstreet, W.P. and Bostian, C.W. (1979) Crosstalk cancellation on linearly and circularly polarized communications satellite link. Radio Sci. 14, 1041-1047.

59) Nouri, M. and Braine, M.R. (1980) Uplink adaptive depolarisation cancellation in a ground-to-satellite link. Electron. Lett. 16, 68-69.

60) Persinger, R.R., Gruner, R.W., Effland, J.E. and DiFonzo, D.F. (1981) Operational measurements of a $4 / 6 \mathrm{GHz}$ adaptive polarization compensation network employing up/down-link correlation algorithm. IEE Conf. Publ. 195 (2), 181-187 (Inst. Elec. Eng., London).

61) McEwan, N.J., Günes, M. and Mahmoud, M.S. (1981) RF methods for adaptive cancellation of cross polarisation in microwave satellite systems. IEE Conf. Publ. 195 (2), 188-192 (Inst. Elec. Eng., London).

62) Yamada, M., Yuki, H., Inagaki, K., Endo, S. and Matsunaka, N. (1982) Compensation techniques of rain depolarization in satellite communications. Radio Sci. 17, 1220-1230.

63) Ulbrich, C.W. (1983) Natural variation in the analytical form of the raindrop size distribution. J. Clim. Appl. Meteorol. 22, 1764-1775.

64) Seliga, T.A. and Bringi, V.N. (1976) Potential use of radar differential reflectivity measurements at orthogonal polarizations for measuring precipitation. J. Appl. Meteorol. 15, 69-76.

65) Seliga, T.A. and Bringi, V.N. (1978) Differential reflectivity and differential phase shift: Application in radar meteorology. Radio Sci. 13, 271-275.

66) Sachidananda, M. and Zrnić, D.S. (1986) Differential propagation phase shift and rainfall rate estimation. Radio Sci. 21, 235-247.

67) Maki, M., Park, S.-G. and Bringi, V.N. (2005) Effect of natural variations in rain drop size distributions on rain rate estimator of $3 \mathrm{~cm}$ wavelength polarimetric radar. J. Meteorol. Soc. Jpn. 83, 871893.

68) Bringi, V.N., Thurai, M., Nakagawa, K., Huang, G.J., Kobayashi, T., Adachi, A. et al. (2006) Rainfall estimation from C-band polarimetric radar in Okinawa, Japan: Comparisons with 2Dvideo disdrometer and $400 \mathrm{MHz}$ wind profiler. J. Meteorol. Soc. Jpn. 84, 705-724.

69) Maki, M., Maesaka, T., Misumi, R., Iwanami, K., Suzuki, S., Kato, A. et al. (2008) X-band polarimetric radar network in the Tokyo metropolitan area-X-NET- The 5th European Conf. on Radar in Meteorol. and Hydrology. (ERAD 2008).

70) National Research Institute for Earth Science and Disaster Prevention (NIED): X Band MultiParameter Radar Rainfall Observation, Rain Attenuation Impact, http://www.bosai.go.jp/ kiban/radar/index_e.htm.

71) Doviak, R.J. and Zrnić, D.S. (1993) Doppler Radar and Weather Observations (2nd ed.). Academic Press, New York.

72) McCormick, G.C. and Hendry, A. (1975) Principles for the radar determination of the polarization properties of precipitation. Radio Sci. 10, 421434.

73) Jameson, A.R. (1985) Microphysical interpretation of multiparameter radar measurements in rain, Part III: interpretation and measurement of propagation differential phase shift between orthogonal linear polarizations. J. Atmos. Sci. 42, 607-614.

74) Tsang, L. (1991) Polarimetric passive microwave 
remote sensing of random discrete scatterers and rough surfaces. J. Electromagn. Waves Appl. 5, $41-57$.

75) Kutuza, B.G., Zagorin, G.K., Hornbostel, A. and Schroth, A. (1998) Physical modeling of passive polarimetric microwave observations of the atmosphere with respect to the third Stokes parameter. Radio Sci. 33, 677-695.

76) Tazaki, T., Tabuchi, H., Ikeda, K., Oguchi, T. and Ito, S. (2000) Laboratory measurements of polarimetric radar signatures of randomly distributed spherical and spheroidal scatterers at $30 \mathrm{GHz}$. IEE Proc., Microw. Antennas Propag. 147, 8-12.

77) Chandrasekhar, S. (1950) Radiative Transfer. Clarendon, London.

78) Furutsu, K. (1982) Wave Propagation in Random Media. Iwanami Shoten, Publishers, Tokyo (in Japanese).

79) Wen, B., Tsang, L., Winebrenner, D.P. and Ishimaru, A. (1990) Dense medium radiative transfer theory: Comparison with experiment and application to microwave remote sensing and polarimetry. IEEE Trans. Geosci. Remote Sens. 28, 46-59.

80) Kuščer, I. and Ribarič, M. (1959) Matrix formalism in the theory of diffusion of light. Opt. Acta (Lond.) 6, 42-51.

81) Sekera, Z. (1966) Scattering matrices and reciprocity relationships for various representations of the state of polarization. J. Opt. Soc. Am. 56, $1732-1740$.

82) Gel'fand, I.M. and Šapiro, Ya. (1956) Representations of the group of rotations in three-dimensional space and their applications. Am. Math. Soc. Transl. 2, 207-316.

83) Burridge, R. (1969) Spherically symmetric differential equations, the rotation group, and tensor spherical functions. Proc. Camb. Philos. Soc. 65, 157-175.

84) Yamanouchi, T. (1957) The Rotation Group and Its Representations. Iwanami Shoten, Publishers, Tokyo (in Japanese).

85) Oguchi, T. (1980) Effect of incoherent scattering on attenuation and cross polarization of millimeter wave due to rain: Preliminary calculations at 34.8 and $82 \mathrm{GHz}$ for spherical raindrops. J. Radio Res. Labs. (Japan) 27, 1-51.

86) Oguchi, T. (1986) Effects of incoherent scattering on attenuation and depolarization of millimeter and optical waves due to hydrometeors. Radio Sci. 4, $717-730$.

87) Ito, S. and Oguchi, T. (1987) An approximate method for solving the vector radiative transfer equation in discrete random media. Radio Sci. 22, 873-879.

88) Ito, S. and Oguchi, T. (1989) Approximate solutions of the vector radiative transfer equation for linearly polarized light in discrete random media. J. Opt. Soc. Am. A 6, 1852-1858.
89) Oguchi, T. (1991) Effects of incoherent scattering on microwave and millimetre wave communications through rain. Electron. Lett. 27, 759-760.

90) Oguchi, T. and Ito, S. (1990) Multiple scattering effects on the transmission and reflection of millimeter pulse waves in rain. Radio Sci. 25, 205-216.

91) Iguchi, T., Meneghini, R. and Kumagai, H. (1992) Radar depolarization signatures of rain in cumulus clouds measured with a dual-frequency airborne radar. Proc. IGARSS'92 Symp., 1728-1730.

92) Ito, S., Oguchi, T., Iguchi, T., Kumagai, H. and Meneghini, R. (1995) Depolarization of radar signals due to multiple scattering in rain. IEEE Trans. Geosci. Remote Sens. 33, 1057-1062.

93) Ito, S., Kobayashi, S. and Oguchi, T. (2007) Multiple-scattering formulation of pulsed beam waves in hydrometeors and its application to millimeter-wave weather radar. IEEE Geosci. Remote Sens. Lett. 4, 13-17.

94) Kobayashi, S., Ito, S., Tanelli, S., Oguchi, T. and Im, E. (2007) A time-dependent multiple scattering theory for a pulsed radar with a finite beamwidth. Radio Sci. 42, RS4001, doi:10.1029/ 2006RS003555.

95) Barabanenkov, Y.N., Kravtsov, Y.A., Ozrin, V.D. and Saichev, A.I. (1991) Enhanced backscattering: The universal wave phenomena. Proc. IEEE 79, 1367-1370.

96) Kuga, Y., Tsang, L. and Ishimaru, A. (1985) Depolarization effects of the enhanced retroreflectance from a dense distribution of spherical particles. JOSA Commun. 2, 616-618.

97) Mandt, C.E., Tsang, L. and Ishimaru, A. (1990) Copolarized and depolarized backscattering enhancement of random discrete scatterers of large size based on second-order ladder and cyclical theory. J. Opt. Soc. Am. A 7, 585-592.

98) Kobayashi, S., Tanelli, S. and Im, E. (2005) Secondorder multiple scattering theory associated with backscattering enhancement for a millimeter wavelength weather radar with a finite beam width. Radio Sci. 40, RS6015, doi:10.1029/ 2004RS003219.

99) Ihara, T., Oguchi, T. and Tazaki, T. (2004) Measurement of backscattering enhancement due to random particles at $30 \mathrm{GHz}$ band using mirror image technique. Proc. URSI Commission F Open Symposium, Union Radio Sci. Int., Cairns, Queensland, Australia, June.

100) Oguchi, T. and Ihara, T. (2006) Computer simulation of enhanced backscattering from randomly distributed spherical scatterers at $30 \mathrm{GHz}$ and comparison with measurement. Radio Sci. 41, RS6002, doi:10.1029/2006RS003468.

(Received Feb. 18, 2010; accepted Apr. 19, 2010) 


\section{Profile}

Sogo Okamura was born in 1918 in Mie, Japan. He graduated from the Department of Electrical Engineering in the Faculty of Engineering at the University of Tokyo in 1940 and received his Doctor of Engineering in 1951 from the same university.

After serving as a full-time Lecturer and an assistant Professor, he held the position of full Professor at the Faculty of Engineering of the University of Tokyo from 1951 until his retirement in 1978 where he worked on research and education in the field of microwave engineering. From 1973 to 1975, he served as Dean of the Faculty of Engineering, and from 1975 to 1977, he served as Special Adviser to the President of the University of Tokyo. In 1978, he was conferred the title of Professor Emeritus. In addition to his professorship at the University of Tokyo, he assumed a concurrent

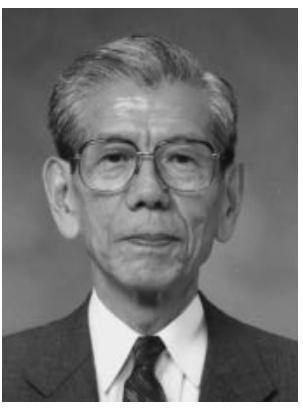
position in 1956 as Chief of the Millimeter Wave Research Section of the Radio Research Laboratory (currently the National Institute of Information and Communications Technology) and initiated research on millimeter-wave propagation in rain in the $35-\mathrm{GHz}$ band.

After retiring from the University of Tokyo in 1978, he became a Professor of Engineering at Tokyo Denki University, and, from 1990 to 1998 he was President of that university. From 1986 to 1994, he served as Senior Adviser to the Rector of the United Nations University. From 1999 to 2003, he was appointed Chairperson of the Board of Directors at the International University of Japan.

He has held important posts in numerous international and/or national organizations, foundations, and incorporated bodies: To name just a few, he has been the Commission-A Chairperson of the International Union of Radio Science (URSI) from 1978 to 1981, and the Vice President of the URSI from 1981 to 1987. He has also been a supervisor and later a member of the board of directors of the Japan Society for the Promotion of Science (JSPS) from 1978, and from 1981 as the Director-General of JSPS. From 1985, he has been a member of the Science Council of Japan (SCJ), from 1988 to 1994, he was the Chairperson of Section 5 of the SCJ, and he was the President of the Engineering Academy of Japan from 1994 to 1998.

His research may be classified into three categories: 1) detailed experimental and theoretical analyses of the characteristics of electron tubes and semiconductor devices, with particular interest in the mutual interaction of electron beams or carriers with their driving circuits; 2) developments in microwave-measurement techniques, with emphasis on power measurements and standards, and 3) atmospheric propagation of millimeter waves that include the effects of rain described in this paper.

He is a member of the Japan Academy, a Life Fellow of the Institute of Electrical and Electronics Engineers, an Honorary Member of the Institute of Electrical Engineers of Japan, an Honorary Member of the Institute of Electronics, Information and Communication Engineers, and the Executive Adviser of the Engineering Academy of Japan.

He has been awarded many prestigious prizes, including the Medal of Honor with a Purple Ribbon in 1984, the Second Order of Merit with the Rising Sun in 1989, and a Person of Cultural Merit in 1991. 


\section{Profile}

Tomohiro Oguchi was born in 1932 in Tokyo, Japan. He graduated from the Department of Electrical Engineering in the Faculty of Engineering at Keio University in 1956 and received a Doctor of Engineering in 1965 from the same university.

In 1956, he joined the Radio Research Laboratory (later the Communications Research Laboratory and that is currently the National Institute of Information and Communications Technology), where he conducted theoretical studies on radio-wave propagation in rain with special emphasis on the effect of polarization under the guidance of Professor Sogo Okamura, who concurrently served as Chief of the Millimeter Wave Research Section of that laboratory. After he retired from the same laboratory in 1991, he was a Faculty Member at the Tokyo Metropolitan Institute of Technology from 1991

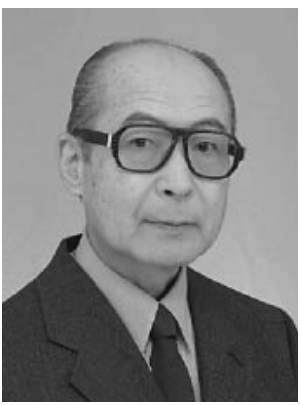
to 1995. In 1995, he became a Faculty Member at Kanto Gakuin University in Yokohama, where he is currently a Researcher with the Institute of Science and Technology. He is concurrently acting as a Guest Researcher with the National Research Institute for Earth Science and Disaster Prevention in Tsukuba. His other former activities include having been a Visiting Research Associate with the ElectroScience Laboratory of Ohio State University in Columbus, Ohio, from 1968 to 1969, a Foreign Associate Editor of Radio Science with the American Geophysical Union from 1980 to 1983, and a Visiting Professor with Wuhan University in Wuhan, China, in 1983.

His research interests include the effects of rain on the propagation and scattering of radio waves, particularly those of drop distortion and multiple scattering, and, more recently the detection by radar of volcanic eruptions.

He is a member of the American Geophysical Union, the Institute of Electrical and Electronics Engineers, and Sigma Xi. In addition to several prestigious national medals he has received, including the Medal of Honor with a Purple Ribbon in 1993 and the Fourth Order of Merit with the Rising Sun in 2002, he was awarded the John Howard Dellinger Gold Medal of the International Union of Radio Science (URSI) in 1996. 